TRANSACTIONS OF THE

AMERICAN MATHEMATICAL SOCIETY

Volume 351, Number 7, Pages 2695-2729

S 0002-9947(99)02230-8

Article electronically published on February 5, 1999

\title{
THE QUANTUM COHOMOLOGY RING OF FLAG VARIETIES
}

\author{
IONUŢ CIOCAN-FONTANINE
}

\begin{abstract}
We describe the small quantum cohomology ring of complete flag varieties by algebro-geometric methods, as presented in our previous work Quantum cohomology of flag varieties (Internat. Math. Res. Notices, no. 6 (1995), 263-277). We also give a geometric proof of the quantum Monk formula.
\end{abstract}

\section{INTRODUCTION}

The quantum cohomology ring of a projective manifold $X$ is a deformation of the usual cohomology ring which has been introduced by physicists (see $[\mathrm{W}]$ ). The quantum multiplication of cohomology classes on $X$ is obtained by adding to the usual cup-product the so-called instanton corrections. These can be in turn interpreted as intersection numbers on a sequence of moduli spaces of (holomorphic) maps $\mathbb{P}^{1} \rightarrow X$. To make this interpretation rigorous according to mathematical standards, one encounters severe problems, mainly because these moduli spaces are not compact and they may have the wrong dimension.

Recently, substantial efforts have been made to put the theory on firm mathematical footing, and the first construction of quantum cohomology rings, using methods of symplectic topology, has been given by Ruan and Tian [RT] for a large class of manifolds (semi-positive symplectic manifolds), which includes projective Fano and Calabi-Yau varieties.

Meanwhile, an algebro-geometric axiomatic approach has been started by Kontsevich and Manin in [KM], and the program outlined there has just been completed, using Kontsevich's notion of stable maps, in the works of Behrend, Behrend and Manin and Li and Tian (see [Beh], [BM], [LT2]). An account for some of the results along these lines can be found in [FP]. Another algebro-geometric approach for the case of homogeneous spaces can be found in [LT1].

There are actually two different deformations of the cohomology ring of $X$ that can be considered; they are called the big quantum cohomology ring and the small quantum cohomology ring in $[\mathrm{FP}]$. The big quantum ring is an algebra over a power series ring and contains most of the interesting data from the point of view of enumerative geometry. The "parameter" space for the deformation is the vector space $H^{*}(X ; \mathbb{C})$ itself. The small quantum ring is obtained by deforming the cohomology classes over $H^{1,1}(X)$ only. For Fano varieties this gives an algebra over a

Received by the editors April 2, 1997.

1991 Mathematics Subject Classification. Primary 14M15; Secondary 14N10.

Key words and phrases. Quantum cohomology, flag varieties, hyperquot schemes, degeneracy loci.

(C)1999 American Mathematical Society 
polynomial ring, while for Calabi-Yau threefolds, which is the case of main interest for physicists, the two rings are essentially the same.

In this paper we shall be concerned only with the small quantum cohomology ring, and from now on we drop the adjective "small" when referring to it.

Computations of quantum cohomology rings have been worked out for several examples: Batyrev [Bat] for toric varieties, Crauder-Miranda [CM] for some Del Pezzo surfaces, Siebert-Tian [ST] for Grassmannians (see also [Be2]), and Qin-Ruan $[\mathrm{QR}]$ for some projective bundles over $\mathbb{P}^{n}$.

In [GK], based on the conjectures of conformal field theory, Givental and Kim conjectured a presentation of the quantum cohomology ring of complete flag varieties. Their computation relied on the assumed existence of an equivariant version of quantum cohomology, satisfying some functorial properties.

Specifically, let $F(n)$ be the variety of complete flags in $\mathbb{C}^{n}$. The classical cohomology ring with $\mathbb{Z}$-coefficients has a presentation given by $\mathbb{Z}\left[x_{1}, \ldots, x_{n}\right] / I$, where $x_{1}, \ldots, x_{n}$ are the Chern classes of some natural line bundles on $F(n)$ and $I=\left(R_{1}(n), R_{2}(n), \ldots, R_{n}(n)\right)$, with $R_{i}(n)$ the $i^{\text {th }}$ elementary symmetric function in $x_{1}, \ldots, x_{n}$. By a general result of Siebert and Tian [ST], the quantum cohomology ring of $F(n)$ will then have a presentation given by

$$
\mathbb{Z}\left[x_{1}, \ldots, x_{n}, q_{1}, \ldots, q_{n-1}\right] / I_{q},
$$

where the new variables $q_{i}$ are the deformation parameter, and $I_{q}$ is an ideal generated by deformations of the generators of $I$. The Givental-Kim conjecture identifies the generators of $I_{q}$.

The present paper is the result of a project whose goals were on the one hand to give an algebro-geometric proof of this conjecture, by building on the ideas in $[\mathrm{Be} 2]$ (see also [Be1] and [BDW]), and on the other hand to develop an analogue in the case of complete flag varieties of the "quantum Schubert calculus," introduced by A. Bertram [Be2] for Grassmannians. In analogy with the classical case, this consists of solving the

Quantum Giambelli problem. Find polynomial representatives for the cosets corresponding to the Schubert classes in the presentation $(*)$.

Quantum Pieri problem. Express the (quantum) product of a "special" Schubert class and a general one in the basis of Schubert classes.

Here special Schubert classes are the Chern classes of the tautological vector bundles on $F(n)$.

The main ideas of this approach were presented in the note [C-F1], together with a proof for the Givental-Kim conjecture. Another result obtained there is the solution of the quantum Giambelli problem for the special Schubert classes.

Using this special case and the (geometrically obvious) nonnegativity of the structure constants for the basis of the quantum cohomology ring consisting of Schubert classes, Fomin, Gelfand, and Postnikov [FGP] were able to give a combinatorial construction of the quantum Schubert polynomials, thus solving the quantum Giambelli problem completely! They also stated and proved the quantum Monk formula, which is a very important case of the quantum Pieri problem. In view of the above, this is a certain identity involving the quantum Schubert polynomials. The proof they give is again combinatorial. 
The starting point of our study is the observation that the 3-point genus 0 Gromov-Witten invariants of $F(n)$, which are the structure constants for the quantum cohomology ring, can be realized as intersection numbers on a certain compactification of the scheme $\operatorname{Hom}\left(\mathbb{P}^{1}, F(n)\right)$. This compactification has been named the hyperquot scheme in [C-F1] and is a natural generalization of Grothendieck's Quot scheme. It was discovered first (although we were not aware of it until very recently) several years ago by G. Laumon [Lau1], [Lau2], in a completely different context.

In this paper we complete, clarify and simplify the geometric approach of [C-F1]. We provide an argument which establishes simultaneously (see Theorem 5.6) both the validity of the Givental-Kim presentation and the special case of the quantum Giambelli formula mentioned above. Although the idea is certainly the same, the proof here is significantly simpler. The second main point of the paper is to give a direct geometric proof of the quantum Monk formula. Both proofs are based on an analysis of the boundary of the compactification, which is much more precise than the one we sketched in [C-F1].

We mention that since [C-F1] appeared, several different proofs (and generalizations) of the Givental-Kim conjecture have been found. First, the equivariant approach of Givental and Kim has been recently completed in [G] and [Ki2], giving in particular an independent proof of the presentation conjectured in [GK]. Yet another proof, which works for all homogeneous spaces of type $G / B$, was given in [Ki3]. Finally, in a May 1996 talk at the University of Washington, D. Peterson announced that he obtained the analogue of the quantum Monk formula for all $G / B$, and he also computed the quantum cohomology ring for all $G / P$ 's. As far as we know, this work has not been written down yet in preprint form.

Acknowledgements. This paper is a rewriting of my Ph.D. thesis [C-F2]. My special gratitude goes to my advisor, Aaron Bertram, for suggesting the problem, and for his continuous help and encouragement. Thanks are also due to William Fulton, whose many comments and suggestions led to substantial improvements of earlier versions of the paper.

\section{Flag VARIETIES AND THEIR COHOMOLOGY}

Let $V$ be a complex $n$-dimensional vector space and define $F=F(V)$ to be the variety parametrizing complete flags of subspaces:

$$
U_{\bullet}:\{0\}=U_{0} \subset U_{1} \subset U_{2} \subset \cdots \subset U_{n-1} \subset U_{n}=V .
$$

Throughout the paper we will let $V_{X}=V \otimes \mathcal{O}_{X}$ be the trivial rank $n$ vector bundle determined by $V$ on a scheme $X$. There is a universal flag of subbundles

$$
E_{1} \subset E_{2} \subset \cdots \subset E_{n-1} \subset E_{n}=V_{F}
$$

and a universal sequence of quotient bundles

$$
V_{F}=L_{n} \rightarrow L_{n-1} \rightarrow \cdots \rightarrow L_{1} \rightarrow 0,
$$

where $L_{i}=V_{F} / E_{n-i}$ for $i=1, \ldots, n-1$.

Fix a complete flag of subspaces $V_{\bullet}:=V_{1} \subset \cdots \subset V_{n-1} \subset V_{n}=V$. Let $S_{n}$ be the symmetric group on $n$ letters. For $w \in S_{n}$, let

$$
r_{w}(q, p)=\operatorname{card}\{i \mid i \leq q, w(i) \leq p\} .
$$


We denote by $\ell(w)$ the length of the permutation $w$ (i.e., the number of inversions in $w)$. The Schubert variety associated to $w$ is defined by

$$
\Omega_{w}=\Omega_{w}\left(V_{\bullet}\right)=\left\{U_{\bullet} \in F \mid \operatorname{rank}_{U}\left(V_{p} \otimes \mathcal{O}_{F} \rightarrow L_{q}\right) \leq r_{w}(q, p), 1 \leq p, q \leq n\right\} .
$$

$\Omega_{w}$ is an irreducible subvariety of $F$, of codimension $\ell(w)$. We will identify as needed each of these subvarieties with the elements determined by their fundamental classes in $H_{*}(F ; \mathbb{Z})$ or, via Poincaré duality, in $H^{*}(F ; \mathbb{Z})$. This should not lead to confusion.

Let $w_{0} \in S_{n}$ be the permutation of longest length, given by

$$
w_{0}(i)=n-i+1, \quad i=1, \ldots, n .
$$

The following results are classical.

Theorem 0.1 (Ehresmann, $[\mathrm{E}]) .\left\{\Omega_{w}\right\}_{w \in S_{n}}$ is an additive basis for $H^{*}(F ; \mathbb{Z})$. Moreover, its Poincaré dual basis is given by $\left\{\Omega_{w_{0} \cdot w}\right\}_{w \in S_{n}}$.

Theorem 0.2 (Borel, $[\mathrm{Bo}])$. Let $x_{i}=c_{1}\left(\operatorname{ker}\left(L_{i} \rightarrow L_{i-1}\right)\right), i=1, \ldots, n$. Then

$$
H^{*}(F ; \mathbb{Z}) \cong \mathbb{Z}\left[x_{1}, x_{2}, \ldots, x_{n}\right] /\left(R_{1}(n), R_{2}(n), \ldots, R_{n}(n)\right),
$$

where $R_{i}(n)$ is the $i^{\text {th }}$ elementary symmetric function in $x_{1}, x_{2}, \ldots, x_{n}$.

To express $\Omega_{w}$ in $H^{*}(F ; \mathbb{Z})$ in terms of the above presentation, we need the Schubert polynomials of Lascoux and Schützenberger [LS1], [LS2]. Define operators $\partial_{i}, i=1, \ldots, n-1$, on $\mathbb{Z}\left[x_{1}, \ldots, x_{n}\right]$ by

$$
\partial_{i} P=\frac{P\left(x_{1}, \ldots, x_{n}\right)-P\left(x_{1}, \ldots, x_{i-1}, x_{i+1}, x_{i}, x_{i+2}, \ldots, x_{n}\right)}{x_{i}-x_{i+1}} .
$$

For any $w \in S_{n}$, write $w=w_{0} \cdot s_{i_{1}} \cdots s_{i_{k}}$, with $k=\frac{n(n-1)}{2}-\ell(w)$, where $s_{i}=(i, i+1)$ is the transposition interchanging $i$ and $i+1$. The polynomial $\mathfrak{S}_{w}(x) \in \mathbb{Z}\left[x_{1}, \ldots, x_{n}\right]$ defined by

$$
\mathfrak{S}_{w}(x)=\partial_{i_{k}} \circ \ldots \circ \partial_{i_{1}}\left(x_{1}^{n-1} x_{2}^{n-2} \ldots x_{n-1}\right)
$$

is the Schubert polynomial associated to $w$. With this definition, we have a Giambelli type formula, due to Bernstein, Gelfand and Gelfand [BGG] and Demazure [D].

Theorem 0.3. $\Omega_{w}=\mathfrak{S}_{w}\left(x_{1}, \ldots, x_{n}\right)$ in $H^{*}(F ; \mathbb{Z})$.

\section{The moduli SPACE of MAPS AND The hyperquot SCheme}

Let $Y_{i}:=\Omega_{w_{0} \cdot s_{i}}$ be the Poincaré dual of $\Omega_{s_{i}}$. By Theorem 0.1, these classes form a basis of $H_{2}(F ; \mathbb{Z})$. Let $f: \mathbb{P}^{1} \rightarrow F$ be a holomorphic map. Since $\mathcal{O}_{F}\left(\Omega_{s_{i}}\right)$ are nef line bundles, $f_{*}\left[\mathbb{P}^{1}\right]=\sum_{i=1}^{n-1} d_{i} Y_{i}$, with $d_{i} \geq 0$. For fixed $\bar{d}=\left(d_{1}, d_{2}, \ldots, d_{n-1}\right)$, let

$$
H_{\bar{d}}:=\operatorname{Hom}_{\bar{d}}\left(\mathbb{P}^{1}, F\right)
$$

be the moduli space of such maps. The usual way to put a structure of quasiprojective scheme on this space is to embed it in the Hilbert scheme of $\mathbb{P}^{1} \times F$, by identifying a map with its graph. Since $F$ is a homogeneous space, its tangent bundle $T_{F}$ is generated by sections, and therefore so is $f^{*} T_{F}$. Hence $H^{1}\left(\mathbb{P}^{1}, f^{*} T_{F}\right)=0$ for every $f: \mathbb{P}^{1} \rightarrow F$. Standard deformation theory implies then that $H_{\bar{d}}$ is smooth, of dimension $h^{0}\left(\mathbb{P}^{1}, f^{*} T_{F}\right)$. It is well known (see e.g. [Mo]) that the canonical class 
of $F$ is given by $K_{F}=-2\left(\Omega_{s_{1}}+\cdots+\Omega_{s_{n-1}}\right)$. From Riemann-Roch it follows that $H_{\bar{d}}$ has dimension

$$
\operatorname{dim} F+f_{*}\left[\mathbb{P}^{1}\right] \cdot\left(-K_{F}\right)=\frac{n(n-1)}{2}+2 \sum_{i=1}^{n-1} d_{i} .
$$

The moduli space of maps comes with a universal "evaluation" morphism

$$
e v: \mathbb{P}^{1} \times H_{\bar{d}} \rightarrow F,
$$

given by $e v(t,[f])=f(t)$, which can be used to pull-back Schubert varieties to $H_{\bar{d}}$. More precisely, for $t \in \mathbb{P}^{1}, w \in S_{n}$, define a subscheme of $H_{\bar{d}}$ by

$$
\Omega_{w}(t)=e v^{-1}\left(\Omega_{w}\right) \cap\left(\{t\} \times H_{\bar{d}}\right) .
$$

Set-theoretically, this pull-back can be described as

$$
\Omega_{w}(t)=\left\{[f] \in H_{\bar{d}} \mid f(t) \in \Omega_{w}\right\} .
$$

The definition of the quantum cohomology ring, which will be made precise in Definition 4.4, involves counting the number of points in zero-dimensional intersections of such subvarieties of $H_{\bar{d}}$, under suitable genericity conditions. Since $H_{\bar{d}}$ is only quasiprojective, to make the counting well-defined we will realize these numbers as intersection numbers on a certain compactification of $H_{\bar{d}}$. This compactification is a "flagged" version of Grothendieck's Quot scheme, and we will discuss first how this scheme appears naturally in our context.

A map $f: \mathbb{P}^{1} \rightarrow F$ with $f_{*}\left[\mathbb{P}^{1}\right]=\sum_{i=1}^{n-1} d_{i} Y_{i}$ induces (by pull-back of the universal sequence of quotients on $F$ ) a flag of quotient bundles

$$
V_{\mathbb{P}^{1}} \rightarrow Q_{n-1} \rightarrow \cdots \rightarrow Q_{1},
$$

with $\operatorname{rank} Q_{i}=i, \operatorname{deg}\left(Q_{i}\right)=d_{i}$, and conversely, every such sequence on $\mathbb{P}^{1}$ comes from a map as above. Hence one can also think of $H_{\bar{d}}$ as being the moduli space of flagged vector bundle quotients of the trivial rank $n$ bundle on $\mathbb{P}^{1}$, with given ranks and degrees, and a compactification is obtained by considering the flat limits for these quotients.

The pull-backs of Schubert varieties defined above can also be described as degeneracy loci by

$\Omega_{w}(t)=\left\{[f] \mid \operatorname{rank}_{[f]}\left(V_{p} \otimes \mathcal{O} \rightarrow e v^{*} L_{q}\right) \leq r_{w}(q, p), 1 \leq p, q \leq n-1\right\} \cap\left(\{t\} \times H_{\bar{d}}\right)$,

and one may try to extend them across the boundary of the compactification by extending $e v^{*} L_{q}$ and using the same degeneracy locus definition. However, the natural extensions of $e v^{*} L_{q}$ are NOT locally free, so this will not work in this form. Fortunately, a slight modification of the above idea will do the job. From the sequence $(\dagger)$, by setting $S_{i}:=Q_{i}^{*}$, one gets a sequence of subbundles

$$
S_{1} \subset \cdots \subset S_{n-1} \subset V_{\mathbb{P}^{1}}^{*},
$$

with $\operatorname{rank} S_{i}=i, \operatorname{deg}\left(S_{i}\right)=-d_{i}$, and which encodes the map $f$. We let now the quotients $V_{\mathbb{P}^{1}}^{*} / S_{i}$ degenerate in flat families to obtain a compactification of $H_{\bar{d}}$. It turns out that on this compactification $\left(e v^{*} L_{q}\right)^{*}$ extend to vector bundles $\mathcal{S}_{q}$, and we will use them in Section 3 to define and study extensions $\bar{\Omega}_{w}(t)$ of the subvarieties $\Omega_{w}(t) \subset\{t\} \times H_{\bar{d}}$.

We now turn to the precise definition of the compactification. 
Let $X$ be a smooth projective variety over an algebraically closed field $k$ and $\mathcal{E}$ be a rank $r$ vector bundle on $X$. For any scheme $S$ over $k$, let $\pi: X \times S \rightarrow X$ be the projection. Fix $2 \leq n \leq r$ and consider the functor

$$
\mathcal{F}_{n}(X, \mathcal{E}):\{\text { Schemes over } k\} \rightarrow\{\text { Sets }\}
$$

given by

$$
\mathcal{F}_{n}(X, \mathcal{E})(S)=\left\{\begin{array}{c}
\text { equivalence classes of flagged quotient sheaves } \\
\pi^{*} \mathcal{E} \rightarrow Q_{n-1} \rightarrow \cdots \rightarrow Q_{1}, \text { which are flat over } S
\end{array}\right\},
$$

and for a morphism $S \stackrel{\varphi}{\longrightarrow} T$,

$$
\mathcal{F}_{n}(X, \mathcal{E})(\varphi)=\text { pull-back by } \varphi .
$$

Here $\pi^{*} \mathcal{E} \rightarrow Q_{n-1} \rightarrow \cdots \rightarrow Q_{1}$ is equivalent to $\pi^{*} \mathcal{E} \rightarrow Q_{n-1}^{\prime} \rightarrow \cdots \rightarrow Q_{1}^{\prime}$ if there are isomorphisms $\theta_{i}: Q_{i} \rightarrow Q_{i}^{\prime}$ such that all the squares commute. $\left(\mathcal{F}_{2}\right.$ is the usual Quot functor.)

Let $\bar{P}=\left(P_{1}(m), \ldots, P_{n-1}(m)\right)$ be numerical polynomials and define the subfunctor $\mathcal{F}_{n, \bar{P}}(X, \mathcal{E})$ by requiring that $\chi\left(X_{s}, Q_{i_{s}}(m)\right)=P_{i}(m)$ for all $s \in S$. Extending the construction of the Quot scheme we have the following

Theorem 1.1. For fixed $\bar{P}(m), \mathcal{F}_{n, \bar{P}}(X, \mathcal{E})$ is represented by a projective scheme.

We will denote this scheme by $\mathcal{H} \mathcal{Q}_{n, \bar{P}}(X, \mathcal{E})$ and refer to it as the hyperquot scheme associated to $X, \mathcal{E}, n$ and $\bar{P}$. In general this scheme may be very complicated, but in the case of interest for our purposes it is well-behaved. More precisely, let $P_{i}(m)=(m+1) i+d_{n-i}$, which is the Hilbert polynomial of a vector bundle of rank $i$ and degree $d_{n-i}$ on $\mathbb{P}^{1}$. Then $\bar{P}$ is determined by $\bar{d}=\left(d_{1}, \ldots, d_{n-1}\right)$ only. Denote the hyperquot scheme associated to $\mathbb{P}^{1}, V_{\mathbb{P}^{1}}^{*}$ and $\bar{d}$ by $\mathcal{H} \mathcal{Q}_{\bar{d}}$.

Theorem 1.2. $\mathcal{H} \mathcal{Q}_{\bar{d}}$ is smooth, irreducible, of dimension $\frac{n(n-1)}{2}+2 \sum_{i=1}^{n-1} d_{i}$, and contains $H_{\bar{d}}$ as an open, dense subscheme.

These two theorems were discovered by G. Laumon ([Lau1]) and rediscovered, independently, in [C-F1] and [Ki1] in the context of quantum cohomology. For completeness, we have included the proofs from [C-F2] in an appendix to this paper.

\section{The STRUCTURE OF THE BOUNDARY}

As a fine moduli space, $\mathcal{H} \mathcal{Q}_{\bar{d}}$ comes equipped with a universal sequence of quotients

$$
V_{\mathbb{P}^{1} \times \mathcal{H} \mathcal{Q}_{\bar{d}}}^{*} \rightarrow \mathcal{T}_{n-1}^{\bar{d}} \rightarrow \cdots \rightarrow \mathcal{T}_{2}^{\bar{d}} \rightarrow \mathcal{T}_{1}^{\bar{d}} \rightarrow 0
$$

on $\mathbb{P}^{1} \times \mathcal{H} \mathcal{Q}_{\bar{d}}$. Let $\mathcal{S}_{i}^{\bar{d}}:=\operatorname{ker}\left\{V_{\mathbb{P}^{1} \times \mathcal{H} \mathcal{Q}_{\bar{d}}}^{*} \rightarrow \mathcal{T}_{n-i}^{\bar{d}}\right\}$.

For $i=1, \ldots, n-1, \mathcal{S}_{i}^{\bar{d}}$ is a vector bundle of rank $i$ and relative degree $-d_{i}$ (this follows from flatness and the fact that $\mathcal{S}_{i}^{\bar{d}}$ is locally free on each fibre of the projection $\left.\mathbb{P}^{1} \times \mathcal{H} \mathcal{Q}_{\bar{d}} \rightarrow \mathcal{H} \mathcal{Q}_{\bar{d}}\right)$. However, the inclusions in the induced sequence

$$
0 \hookrightarrow \mathcal{S}_{1}^{\bar{d}} \hookrightarrow \mathcal{S}_{2}^{\bar{d}} \hookrightarrow \cdots \hookrightarrow \mathcal{S}_{n-1}^{\bar{d}} \hookrightarrow V_{\mathbb{P}^{1} \times \mathcal{H}_{\bar{d}}}
$$

are injective as maps of sheaves only! 
Obviously, $H_{\bar{d}}$ is the largest subscheme of $\mathcal{H} \mathcal{Q}_{\bar{d}}$ with the property that on $\mathbb{P}^{1} \times H_{\bar{d}}$ all the inclusions are injections of vector bundles. In this section we will describe in detail the loci where these maps degenerate.

We start with a simple lemma, describing the possible ranks for the fibres of the quotient sheaves. For a sheaf $\mathcal{F}$ on a scheme $X$, we will denote the rank of the fibre $\mathcal{F}(x):=\mathcal{F} \otimes k(x)$ at a point $x \in X$ by $\operatorname{rank}_{x}(\mathcal{F})$.

Lemma 2.1. Assume we are given a sequence of vector bundles and sheaf inclusions on $\mathbb{P}^{1}$ :

$$
S_{1} \hookrightarrow \cdots \hookrightarrow S_{n-1} \hookrightarrow V_{\mathbb{P}^{1}}^{*}
$$

with $\operatorname{rank} S_{i}=i$ and $\operatorname{deg} S_{i}=-d_{i}$. Denote by $T_{n-i}$ the quotient $V_{\mathbb{P}^{1}}^{*} / S_{i}$. Let $t \in \mathbb{P}^{1}$ be a point, and define nonnegative integers $e_{i}$ by $\operatorname{rank}_{t}\left(T_{n-i}\right)=n-i+e_{i}$. Then

(2.1.1) $e_{i} \leq \min \left(i, d_{i}\right)$, for $1 \leq i \leq n-1$,

(2.1.2) $e_{i}-e_{i-1} \leq 1$, for $2 \leq i \leq n-1$.

Proof. Since there are sheaf surjections $T_{n-(i-1)} \rightarrow T_{n-i}$, one has $\operatorname{rank}_{t}\left(T_{n-(i-1)}\right) \geq$ $\operatorname{rank}_{t}\left(T_{n-i}\right)$, which gives (2.1.2).

To prove (2.1.1), note first that $e_{i} \leq i$, since $T_{n-i}$ is a quotient of $V_{\mathbb{P 1}}^{*}$. Next, consider the dual map $V_{\mathbb{P}^{1}} \rightarrow S_{i}^{*}$. By assumption, its cokernel $N_{i}$ has rank $e_{i}$ at $t$. Look at the surjection

$$
S_{i}^{*} \rightarrow N_{i}(t) \rightarrow 0
$$

If we let $M_{i}$ denote the kernel, then $\operatorname{deg} M_{i}=d_{i}-e_{i}$. Since $V_{\mathbb{P}^{1}} \rightarrow S_{i}^{*}$ factors through $M_{i}$, the degree of $M_{i}$ is nonnegative, and we get $e_{i} \leq d_{i}$.

Construction 2.2. Let $\bar{e}=\left(e_{1}, \ldots, e_{n-1}\right)$ be a multiindex satisfying the conditions (2.1.1) and (2.1.2) of the lemma. Consider on $\mathbb{P}^{1} \times \mathcal{H} \mathcal{Q}_{\bar{d}-\bar{e}}$ the universal sequence

$$
0 \hookrightarrow \mathcal{S}_{1}^{\bar{d}-\bar{e}} \hookrightarrow \mathcal{S}_{2}^{\bar{d}-\bar{e}} \hookrightarrow \cdots \hookrightarrow \mathcal{S}_{n-1}^{\bar{d}-\bar{e}} \hookrightarrow V_{\mathbb{P}^{1} \times \mathcal{H} \mathcal{Q}_{\bar{d}-\bar{e}}}
$$

For each $1 \leq i \leq n-1$, let

$$
\pi_{i}: X_{i} \longrightarrow \mathbb{P}^{1} \times \mathcal{H} \mathcal{Q}_{\bar{d}-\bar{e}}
$$

be the Grassmann bundle of $e_{i}$-dimensional quotients of $\mathcal{S}_{i}^{\bar{d}-\bar{e}}$. Let $X_{\bar{e}}$ be the fibre product of the $X_{i}{ }^{\prime}$ 's over $\mathbb{P}^{1} \times \mathcal{H} \mathcal{Q}_{\bar{d}-\bar{e}}$, with natural projection $\pi: X_{\bar{e}} \longrightarrow$ $\mathbb{P}^{1} \times \mathcal{H} \mathcal{Q}_{\bar{d}-\bar{e}}$. In what follows, we will use the same notation for the bundles living on $X_{i}$ and their pull-backs to $X_{\bar{e}}$ via the various natural projections. Let

$$
0 \longrightarrow K_{i} \longrightarrow \pi_{i}^{*} \mathcal{S}_{i}^{\bar{d}-\bar{e}} \longrightarrow Q_{i} \longrightarrow 0
$$

be the universal sequence on $X_{i} . K_{i}$ and $Q_{i}$ are vector bundles of ranks $i-e_{i}$ and $e_{i}$, respectively. On $X_{\bar{e}}$ we have then the following diagram: 


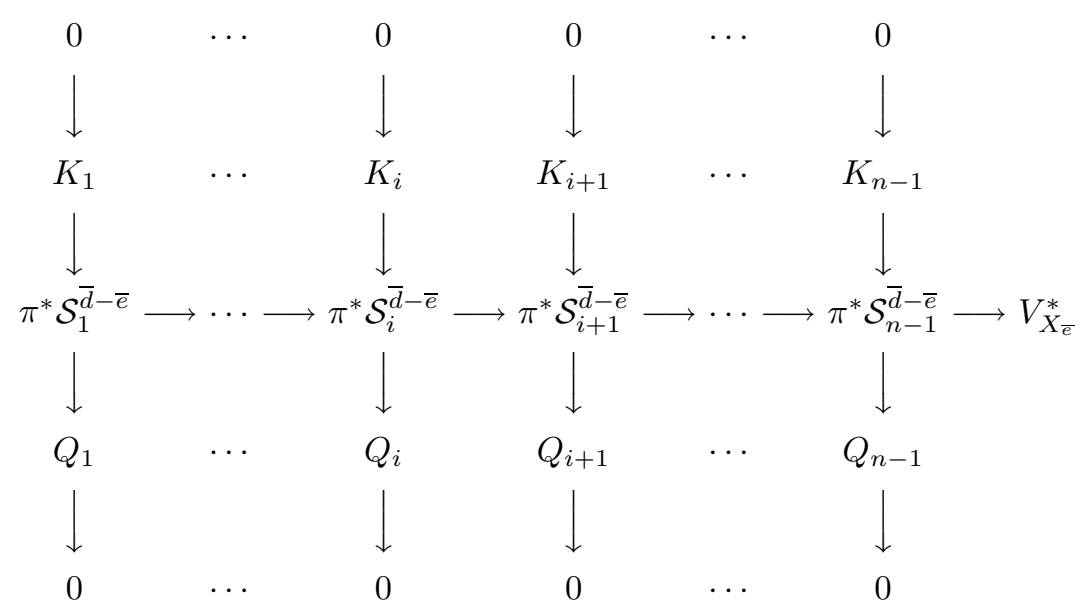

Let $\mathcal{U}_{\bar{e}}$ be the locally-closed subscheme of $X_{\bar{e}}$ determined by the closed conditions

$$
\operatorname{rank}\left(K_{i} \longrightarrow Q_{i+1}\right)=0, \quad \text { for } i=1, \ldots, n-2,
$$

and the open conditions

$$
\operatorname{rank}\left(K_{i} \longrightarrow V_{X_{\bar{e}}}^{*}\right)=i-e_{i}, \quad \text { for } i=1, \ldots, n-1 .
$$

Theorem 2.3. (i) $\mathcal{U}_{\bar{e}}$ is smooth, irreducible, and is of dimension

$$
1+\frac{n(n-1)}{2}+2 \sum_{i=1}^{n-1} d_{i}-\sum_{i=1}^{n-1} e_{i}\left(1+e_{i}-e_{i-1}\right),
$$

where we set, once and for all, $e_{0}=0$. The projection $\pi: \mathcal{U}_{\bar{e}} \rightarrow \mathbb{P}^{1} \times \mathcal{H} \mathcal{Q}_{\bar{d}-\bar{e}}$ is smooth and proper, has irreducible fibres, and its image contains $\mathbb{P}^{1} \times H_{\bar{d}-\bar{e}}$.

(ii) There exist morphisms $h_{\bar{e}}: \mathcal{U}_{\bar{e}} \longrightarrow \mathcal{H} \mathcal{Q}_{\bar{d}}$ with the following properties:

(a) If $\operatorname{rank}_{(t, x)} \mathcal{T}_{n-i}^{\bar{d}}=n-i+e_{i}$ for every $1 \leq i \leq n-1$ at a point $(t, x) \in$ $\mathbb{P}^{1} \times \mathcal{H} \mathcal{Q}_{\bar{d}}$, then $x \in h_{\bar{e}}\left(\mathcal{U}_{\bar{e}}\right)$. In particular $\mathcal{H} \mathcal{Q}_{\bar{d}} \backslash H_{\bar{d}}$ is covered by the union of $h_{\bar{e}}\left(\mathcal{U}_{\bar{e}}\right)$.

(b) The restriction of $h_{\bar{e}}$ to $\pi^{-1}\left(\mathbb{P}^{1} \times H_{\bar{d}-\bar{e}}\right)$ is an isomorphism onto its image.

Proof. (i) We will make use of the following observation.

Let $E$ be a vector bundle of rank $r$ on a smooth variety $Y$, with a subbundle $S$ of rank $s \leq r-k$. Let $p: \check{G}_{k}(E) \rightarrow Y$ be the Grassmann bundle of $k$-dimensional quotients of $E$, with universal quotient bundle $p^{*} E \rightarrow Q$, and let $\Omega(S)$ be the subscheme of $\breve{G}_{k}(E)$ determined by the vanishing of the map $p^{*} S \rightarrow Q$. Then $\Omega(S)$ can be identified with the Grassmann bundle of $k$-dimensional quotients of $E / S$ over $Y$. In particular it is smooth, of pure codimension $k s$ in $\check{G}_{k}(E)$, and $p: \Omega(S) \rightarrow Y$ is smooth, with irreducible fibres.

We now prove by induction on $i$ the following (more general)

Claim 2.4. Let $U_{i} \subset X_{1} \times_{\mathbb{P}^{1} \times \mathcal{H}} \cdots \times_{\mathbb{P}^{1} \times \mathcal{H} \mathcal{Q}} X_{i+1}$ be the locally closed subscheme determined by the conditions

$$
\operatorname{rank}\left(K_{j} \rightarrow V^{*}\right)=j, \quad j=1, \ldots, i+1,
$$

and

$$
\operatorname{rank}\left(K_{j} \rightarrow Q_{j+1}\right)=0, \quad j=1, \ldots, i .
$$


Then $U_{i}$ is smooth, of pure codimension $\sum_{j=1}^{i} e_{j+1}\left(j-e_{j}\right)$ in $X_{1} \times_{\mathbb{P}^{1} \times \mathcal{H}} \cdots \times_{\mathbb{P}^{1} \times \mathcal{H} \mathcal{Q}}$ $X_{i+1}$. The projection $\varrho_{i}: U_{i} \rightarrow \mathbb{P}^{1} \times \mathcal{H} \mathcal{Q}_{\bar{d}-\bar{e}}$ is smooth, with irreducible fibres, and its image contains $\mathbb{P}^{1} \times H_{\bar{d}-\bar{e}}$.

Proof. In the case $i=0, U_{0}$ is either $\mathbb{P}^{1} \times \mathcal{H} \mathcal{Q}_{\bar{d}-\bar{e}}$, if $e_{1}=1$, or the open subscheme of $\mathbb{P}^{1} \times \mathcal{H} \mathcal{Q}_{\bar{d}-\bar{e}}$ where the map $\mathcal{S}_{1}^{\bar{d}-\bar{e}} \rightarrow V^{*}$ is nondegenerate, if $e_{1}=0$. In both cases the claim is clearly true.

Assume that 2.4 is true for $U_{i-1}$. Let $p: \check{G}_{e_{i+1}} \rightarrow U_{i-1}$ be the Grassmann bundle over $U_{i-1}$, obtained by restricting the projection

$$
X_{1} \times_{\mathbb{P}^{1} \times \mathcal{H} \mathcal{Q}} \cdots \times_{\mathbb{P}^{1} \times \mathcal{H} \mathcal{Q}} X_{i+1} \rightarrow X_{1} \times_{\mathbb{P}^{1} \times \mathcal{H} \mathcal{Q}} \cdots \times_{\mathbb{P}^{1} \times \mathcal{H} \mathcal{Q}} X_{i} .
$$

$U_{i}$ is the intersection of the Schubert variety in $\check{G}_{e_{i+1}}$ determined by the vanishing of the map $p^{*} K_{i} \rightarrow Q_{i+1}$ and the open subscheme of $\check{G}_{e_{i+1}}$ given by $\operatorname{rank}\left(K_{i+1} \rightarrow\right.$ $\left.V^{*}\right)=i+1-e_{i+1}$. It follows from the above observation and the induction hypothesis that $U_{i}$ has the claimed codimension, is smooth, and $\varrho_{i}=\varrho_{i-1} \circ p$ is smooth, with irreducible fibres. This completes the proof of 2.4 .

Because $U_{n-2}=\mathcal{U}_{\bar{e}}$, it follows from 2.4 that $\mathcal{U}_{\bar{e}}$ has pure codimension $\sum_{i=1}^{n-2} e_{i+1}\left(i-e_{i}\right)$ in $X_{\bar{e}}$. A simple computation shows that the dimension of $\mathcal{U}_{\bar{e}}$ is given by the formula in the theorem.

(ii) Consider the map

$$
\psi: \mathbb{P}^{1} \times \mathcal{U}_{\bar{e}} \longrightarrow \mathbb{P}^{1} \times \mathbb{P}^{1} \times \mathcal{H} \mathcal{Q}_{\bar{d}-\bar{e}},
$$

$\psi=(i d, \pi)$. Let $\Delta \subset \mathbb{P}^{1} \times \mathbb{P}^{1}$ be the diagonal and let $\tilde{\Delta} \subset \mathbb{P}^{1} \times \mathcal{U}_{\bar{e}}$ be the preimage of $\Delta \times \mathcal{H}_{\mathcal{Q}_{\bar{d}}-\bar{e}}$ via $\psi$. Let $p: \mathbb{P}^{1} \times \mathcal{U}_{\bar{e}} \rightarrow \mathcal{U}_{\bar{e}}$ be the projection. For every $1 \leq i \leq n-1$, define $\tilde{\mathcal{S}}_{i}^{\bar{e}}$ to be the kernel of the natural map

$$
\left.\psi^{*} \mathcal{S}_{i}^{\bar{d}-\bar{e}} \longrightarrow p^{*} Q_{i}\right|_{\tilde{\Delta}}
$$

Since $\tilde{\Delta}$ is a divisor intersecting each fibre of the projection $p$ in exactly one point, and $\left.p^{*} Q_{i}\right|_{\tilde{\Delta}}$ is locally free of rank $e_{i}$ on $\tilde{\Delta}$, it follows that $\tilde{\mathcal{S}}_{i}^{\bar{e}}$ is a vector bundle of rank $i$ and relative degree $-d_{i}$ on $\mathbb{P}^{1} \times \mathcal{U}_{\bar{e}}$. By [H, III.9.9] $\tilde{\mathcal{S}}_{i}^{\bar{e}}$ is therefore flat over $\mathcal{U}_{\bar{e}}$. Via its inclusion in $\psi^{*} \mathcal{S}_{i}^{\bar{d}-\bar{e}}$, this new bundle comes with a sheaf injection $\tilde{\mathcal{S}}_{i}^{\bar{e}} \hookrightarrow$ $V_{\mathbb{P}^{1} \times \mathcal{U}_{\bar{e}}}^{*}$. Moreover, by the construction of $\mathcal{U}_{\bar{e}}$, there are induced sheaf injections

$$
\tilde{\mathcal{S}}_{i}^{\bar{e}} \hookrightarrow \tilde{\mathcal{S}}_{i+1}^{\bar{e}}, i=1, \ldots, n-2, \text { and } \tilde{\mathcal{S}}_{n-1}^{\bar{e}} \hookrightarrow V_{\mathbb{P}^{1} \times \mathcal{U}_{\bar{e}}}^{*},
$$

making the following diagram commutative:

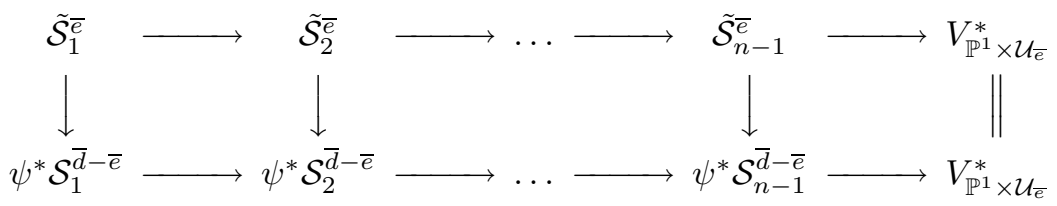

In fact, (2.2.1) is precisely the condition needed for the map $\tilde{\mathcal{S}}_{i}^{\bar{e}} \hookrightarrow V_{\mathbb{P}^{1}}^{*} \mathcal{U}_{\bar{e}}$ to factor through $\tilde{\mathcal{S}}_{i+1}^{\bar{e}}$.

Since $\mathcal{H} \mathcal{Q}_{\bar{d}}$ represents the hyperquot functor, there exists a map

$$
h_{\bar{e}}: \mathcal{U}_{\bar{e}} \longrightarrow \mathcal{H} \mathcal{Q}_{\bar{d}},
$$

such that $\left(i d, h_{\bar{e}}\right)^{*} \mathcal{S}_{i}^{\bar{d}}=\tilde{\mathcal{S}}_{i}^{\bar{e}}$, for every $i=1, \ldots, n-1$. 
To prove $(a)$, let $x \in \mathcal{H} \mathcal{Q}_{\bar{d}}$ be a point, represented by a sequence of vector bundles, together with sheaf injections

$$
S_{1} \hookrightarrow \cdots \hookrightarrow S_{n-1} \hookrightarrow V_{\mathbb{P}^{1}}^{*},
$$

such that $\operatorname{rank}\left(S_{i}\right)=i, \operatorname{deg}\left(S_{i}\right)=-d_{i}$. Assume that $\operatorname{rank}_{t}\left(T_{n-i}\right)=n-i+e_{i}$, where $t \in \mathbb{P}^{1}$ and $T_{n-i}=V_{\mathbb{P}^{1}}^{*} / S_{i}$. To get a point in $\mathcal{U}_{\bar{e}}$ whose image under $h_{\bar{e}}$ is $x$, we need to construct a sequence of bundles

$$
E_{1} \hookrightarrow \cdots \hookrightarrow E_{n-1} \hookrightarrow V_{\mathbb{P}^{1}}^{*},
$$

together with rank $e_{i}$ quotients of the fibres at $t$

$$
E_{i}(t) \rightarrow Q_{i}(t) \rightarrow 0,
$$

satisfying the following conditions:

(2.3.1) $\operatorname{rank}\left(E_{i}\right)=i$ and $\operatorname{deg}\left(E_{i}\right)=-\left(d_{i}-e_{i}\right)$;

(2.3.2) if $K_{i}(t):=\operatorname{ker}\left(E_{i}(t) \rightarrow Q_{i}(t)\right)$, the induced map of vector spaces $K_{i}(t) \rightarrow$ $V_{\mathbb{P}^{1}}^{*}(t)$ is injective;

(2.3.3) $S_{i}=\operatorname{ker}\left(E_{i} \rightarrow Q_{i}(t)\right)$ and all the squares

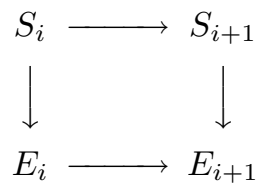

commute (in particular, this implies that $K_{i}(t) \rightarrow Q_{i+1}(t)$ vanishes).

Consider the dual sequence

$$
V_{\mathbb{P}^{1}} \rightarrow S_{n-1}^{*} \rightarrow \cdots \rightarrow S_{1}^{*}
$$

and let $N_{i}$ be the cokernel of the map $V_{\mathbb{P}^{1}} \rightarrow S_{i}^{*}$. Let $M_{i}$ be the kernel of the induced quotient $S_{i}^{*} \rightarrow N_{i}(t)$. By assumption, $N_{i}(t) \cong \mathbb{C}^{e_{i}}$, hence $M_{i}$ is a vector bundle of rank $i$ and degree $d_{i}-e_{i}$ on $\mathbb{P}^{1}$. Since there are natural induced maps $N_{i} \rightarrow N_{i-1}$, we have the following commutative diagram:

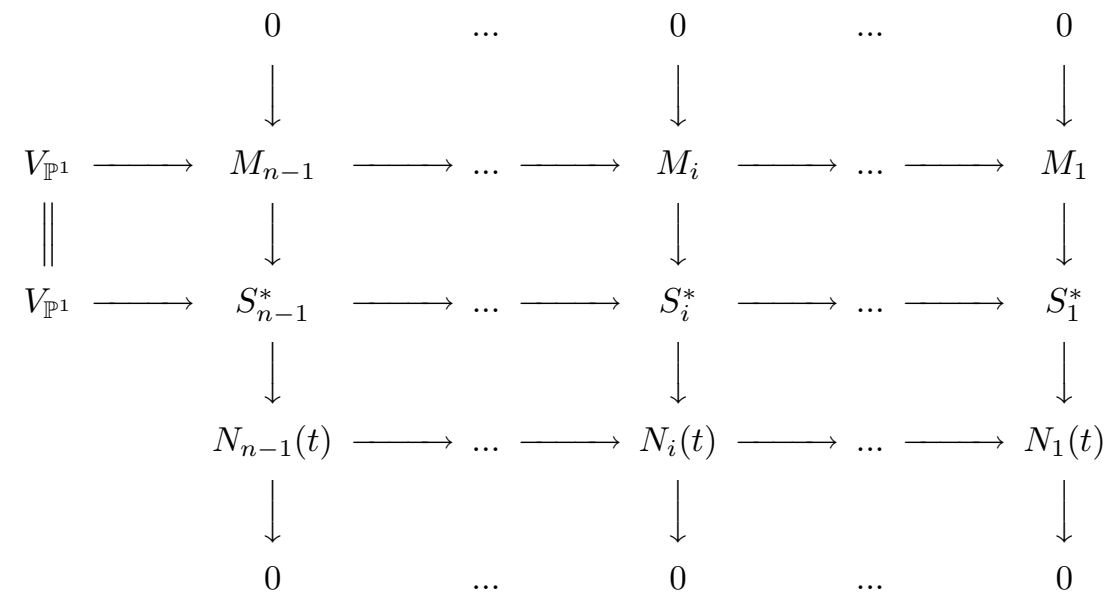

Set $E_{i}:=M_{i}^{*}$ and $Q_{i}(t):=\operatorname{coker}\left(S_{i} \rightarrow E_{i}\right)$. It is straightforward to check that the required conditions are satisfied. Indeed, (2.3.1) and (2.3.3) are obvious from the definition of the bundles $E_{i}$. Moreover, by construction the images of $K_{i}(t)$ and $S_{i}(t)$ in $V^{*}$ coincide. By assumption, the latter image has rank $i-e_{i}$, hence (2.3.2) holds as well. 
Notice that if for every $1 \leq i \leq n-1$ the subscheme of $\mathbb{P}^{1}$ where the sheaf $T_{n-i}$ has rank $n-i+e_{i}$ is the reduced point $t \in \mathbb{P}^{1}$, and the rank is $n-i$ everywhere else, then the sequence

$$
V_{\mathbb{P}^{1}} \rightarrow M_{n-1} \rightarrow \cdots \rightarrow M_{1}
$$

is uniquely determined, and all the maps are surjective. Therefore $h_{\bar{e}}$ is 1 -to- 1 on $\pi^{-1}\left(\mathbb{P}^{1} \times H_{\bar{d}-\bar{e}}\right)$, which gives (b) set-theoretically. By globalizing this observation in exactly the same way as in [Be2, Thm. 3.1], one constructs an inverse to $h_{\bar{e}}$ on the image of $\pi^{-1}\left(\mathbb{P}^{1} \times H_{\bar{d}-\bar{e}}\right)$, so we obtain $(b)$.

Remark 2.5. Denote by $\bar{e}_{i}$ the multiindex $(0, \ldots, 0,1,0, \ldots, 0)$ (with 1 in the $i$ th spot). It follows from Construction 2.2 and the proof of Theorem 2.3 that $\pi: \mathcal{U}_{\bar{e}_{i}} \rightarrow$ $\mathbb{P}^{1} \times \mathcal{H} \mathcal{Q}_{\bar{d}-\bar{e}_{i}}$ is an open immersion. The images $D_{i}:=h_{\bar{e}_{i}}\left(\mathcal{U}_{\bar{e}_{i}}\right)$ are birational to $\mathbb{P}^{1} \times \mathcal{H} \mathcal{Q}_{\bar{d}-\bar{e}_{i}}$. In particular, each $D_{i}$ has codimension 1 in $\mathcal{H} \mathcal{Q}_{\bar{d}}$.

Example 2.6. Let $n=3$ and $\bar{d}=(1,1)$. In this case the boundary consists of the two codimension 1 strata, $D_{1}$ and $D_{2}$, and a codimension 2 stratum $E:=$ $h_{(1,1)}\left(\mathcal{U}_{(1,1)}\right)$.

Since $\mathcal{H} \mathcal{Q}_{(0,0)} \cong H_{(0,0)} \cong F$, it follows that $\mathcal{U}_{(1,1)}=X_{(1,1)}$ is the $\mathbb{P}^{1}$-bundle $\check{\mathbb{P}}\left(\mathcal{S}_{2}^{(0,0)}\right) \stackrel{\pi}{\rightarrow} \mathbb{P}^{1} \times F$, the map $h_{(1,1)}: \mathcal{U}_{(1,1)} \rightarrow E$ is an isomorphism, $K_{1}=0$, and $K_{2}$ is the kernel of the natural surjection

$$
\pi^{*} \mathcal{S}_{2}^{(0,0)} \rightarrow \mathcal{O}_{E}(1) \rightarrow 0 .
$$

Remark 2.7. A somewhat different stratification of $\mathcal{H} \mathcal{Q}_{\bar{d}}$ is given in [Lau2] and is studied further in the recent paper [Kuz].

\section{Schubert Classes on $\mathcal{H} \mathcal{Q}_{\bar{d}}$ And their Restrictions to the boundary}

Recall from Section 1 the universal evaluation morphism ev $: \mathbb{P}^{1} \times H_{\bar{d}} \rightarrow F$ and the subschemes of $H_{\bar{d}}$ :

$$
\Omega_{w}(t)=e v^{-1}\left(\Omega_{w}\right) \cap\left(\{t\} \times H_{\bar{d}}\right), \quad t \in \mathbb{P}^{1}, \quad w \in S_{n} .
$$

To extend these $\Omega_{w}(t)$ to the hyperquot scheme, consider on $\mathbb{P}^{1} \times \mathcal{H} \mathcal{Q}_{\bar{d}}$ the dual universal sequence $V_{\mathbb{P}^{1} \times \mathcal{H} \mathcal{Q}_{\bar{d}}} \rightarrow \mathcal{S}_{n-1}^{*} \rightarrow \cdots \rightarrow \mathcal{S}_{1}^{*}$ and the fixed flag $0=V_{0} \subset V_{1} \subset$ $\cdots \subset V_{n-1} \subset V_{n}=V$. We define $\bar{\Omega}_{w}(t)$ as the restriction to $\{t\} \times \mathcal{H} \mathcal{Q}_{\bar{d}}$ of the appropriate degeneracy locus on $\mathbb{P}^{1} \times \mathcal{H} \mathcal{Q}_{\bar{d}}$ :

$$
\bar{\Omega}_{w}(t):=\left\{\operatorname{rank}\left(V_{p} \otimes \mathcal{O} \rightarrow \mathcal{S}_{q}^{*}\right) \leq r_{w}(q, p), 1 \leq p, q \leq n\right\} \cap\{t\} \times \mathcal{H} \mathcal{Q}_{\bar{d}} .
$$

Obviously, $\Omega_{w}(t) \subset \bar{\Omega}_{w}(t)$. Via the identification $\{t\} \times \mathcal{H} \mathcal{Q}_{\bar{d}} \cong \mathcal{H} \mathcal{Q}_{\bar{d}}$, the subscheme $\bar{\Omega}_{w}(t)$ determines a class in the Chow ring $C H^{*}\left(\mathcal{H} \mathcal{Q}_{\bar{d}}\right)$. We will prove in the next section some "general position" results showing that these classes have the expected codimension and are independent of the choices made in the definition (see Theorem 4.1 and its corollaries). In order to do this, we need to understand first the restriction of $\bar{\Omega}_{w}(t)$ to the boundary.

We keep the notation introduced in Section 2. Let $\bar{e}=\left(e_{1}, \ldots, e_{n-1}\right)$ be a multiindex satisfying the conditions (2.1.1) and (2.1.2) of Lemma 2.1, let $\pi: \mathcal{U}_{\bar{e}} \rightarrow$ $\mathbb{P}^{1} \times \mathcal{H} \mathcal{Q}_{\bar{d}-\bar{e}}$ be the projection, and let $h_{\bar{e}}: \mathcal{U}_{\bar{e}} \rightarrow \mathcal{H} \mathcal{Q}_{\bar{d}}$ be the map defined in Theorem 2.3. 
Lemma 3.1.

$$
h_{\bar{e}}^{-1}\left(\bar{\Omega}_{w}(t)\right)=\pi^{-1}\left(\mathbb{P}^{1} \times \bar{\Omega}_{w}(t)\right) \cup \tilde{\Omega}_{w}^{\bar{e}}(t),
$$

$\tilde{\Omega}_{w}^{\bar{e}}(t)$ being the degeneracy locus inside $\pi^{-1}\left(\{t\} \times \mathcal{H} \mathcal{Q}_{\bar{d}-\bar{e}}\right)$ given by

$$
\operatorname{rank}\left(V_{p} \otimes \mathcal{O} \rightarrow K_{q}^{*}\right) \leq r_{w}(q, p), \quad p, q=1, \ldots, n .
$$

Proof. We reconsider the construction in the proof of Theorem 2.3, (ii), keeping the same notation. Since $\left(i d, h_{\bar{e}}\right)^{*} \mathcal{S}_{q}^{\bar{d}}=\tilde{\mathcal{S}}_{q}^{\bar{e}}$ for every $q=1, \ldots, n-1$, it follows that

$$
h_{\bar{e}}^{-1}\left(\bar{\Omega}_{w}(t)\right)=\left\{y \in \mathcal{U}_{\bar{e}} \mid \operatorname{rank}_{(t, y)} V_{p} \otimes \mathcal{O} \rightarrow\left(\tilde{\mathcal{S}}_{q}^{\bar{e}}\right)^{*} \leq r_{w}(q, p) \text {, for all } p, q\right\},
$$

where $V_{p} \otimes \mathcal{O} \rightarrow\left(\tilde{\mathcal{S}}_{q}^{\bar{e}}\right)^{*}$ is obtained as the composition

$$
V_{p} \otimes \mathcal{O} \rightarrow V \otimes \mathcal{O} \rightarrow\left(\psi^{*} \mathcal{S}_{q}^{\bar{d}-\bar{e}}\right)^{*} \longrightarrow\left(\tilde{\mathcal{S}}_{q}^{\bar{e}}\right)^{*}
$$

with $\psi=(i d, \pi): \mathbb{P}^{1} \times \mathcal{U}_{\bar{e}} \rightarrow \mathbb{P}^{1} \times \mathbb{P}^{1} \times \mathcal{H} \mathcal{Q}_{\bar{d}-\bar{e}}$. The map $\left(\psi^{*} \mathcal{S}_{q}^{\bar{d}-\bar{e}}\right)^{*} \longrightarrow\left(\tilde{\mathcal{S}}_{q}^{\bar{e}}\right)^{*}$ is an isomorphism outside $\tilde{\Delta}$, while its restriction to $\tilde{\Delta}$ factors through $K_{q}^{*}$, and the lemma follows.

We would like now to estimate the codimension of $\tilde{\Omega}_{w}^{\bar{e}}(t)$ in $\mathcal{U}_{\bar{e}}$. The problem is that since $\operatorname{rank}\left(K_{q}^{*}\right)=q-e_{q}$, some of the rank conditions in $\left(^{*}\right)$ may become irrelevant. To deal with this we need some facts about degeneracy loci associated to permutations. The following definitions and results are taken from [F1].

Let $w \in S_{n}$ be a permutation. The following subset of $\{1, \ldots, n\} \times\{1, \ldots, n\}$ is called the essential set associated to $w$ :

$$
\mathcal{E} s s(w)=\left\{(q, p) \mid w(q)>p, w(q+1) \leq p, w^{-1}(p)>q, w^{-1}(p+1) \leq q\right\} .
$$

Assume that on an arbitrary scheme $X$ we are given a morphism of filtered vector bundles

$$
E_{1} \subset \cdots \subset E_{n-1} \subset E_{n} \stackrel{h}{\rightarrow} F_{n} \rightarrow F_{n-1} \rightarrow \cdots \rightarrow F_{1}
$$

with $\operatorname{rank}\left(E_{i}\right)=\operatorname{rank}\left(F_{i}\right)=i$. We consider the subscheme $D_{w}=D_{w}(h)$ defined by

$$
\operatorname{rank}\left(E_{p} \rightarrow F_{q}\right) \leq r_{w}(q, p), \quad 1 \leq p, q \leq n .
$$

This subscheme can be given however using a more economical set of rank conditions.

Proposition 3.2 (Fulton, [F1, Prop. 4.2]). The scheme $D_{w}$ is defined by

$$
\operatorname{rank}\left(E_{p} \rightarrow F_{q}\right) \leq r_{w}(q, p),
$$

for all $p, q \in\{1, \ldots, n\}$ such that $(q, p) \in \mathcal{E} s s(w)$.

Assume now that we are given integers $m$ and $n$ together with sequences

$$
1 \leq m_{1}<\cdots<m_{l} \leq m, \quad 1 \leq n_{1}<\cdots<n_{k} \leq n,
$$

and a collection of nonnegative integers $\left(r_{j, i}\right)$ for $1 \leq j \leq k, 1 \leq i \leq l$.

Definition 3.3. $\left(r_{j, i}\right)$ is said to be a permissible collection of rank numbers if there exists a permutation $w$ in some $S_{h}$, with $h \geq \max \left(n_{k}, m_{l}\right)$, such that

$$
\mathcal{E} s s(w) \subset\left\{m_{1} \ldots, m_{l}\right\} \times\left\{n_{1}, \ldots, n_{k}\right\}
$$


and

$$
r_{j, i}=r_{w}\left(n_{j}, m_{i}\right), \quad \text { for all } 1 \leq j \leq k, \quad 1 \leq i \leq l .
$$

Fulton proves that such $w$, if it exists, is unique up to the embeddings of $S_{h}$ in $S_{h+1}$.

Let $V$ be our fixed $n$-dimensional complex vector space and let $F\left(n_{1}, \ldots, n_{k}\right)$ be the partial flag variety parametrizing sequences of quotients of $V$ with ranks given by the $n_{i}$. Let

$$
V \otimes \mathcal{O} \rightarrow Q_{n_{k}} \rightarrow \cdots \rightarrow Q_{n_{1}}
$$

be the universal quotient sequence on $F\left(n_{1}, \ldots, n_{k}\right)$.

For any collection of nonnegative integers $\mathbf{r}=\left(r_{j, i}\right), 1 \leq j \leq k, 1 \leq i \leq n$, let $D_{\mathbf{r}} \subset F\left(n_{1}, \ldots, n_{k}\right)$ be the degeneracy locus defined by the conditions

$$
\operatorname{rank}\left(V_{i} \longrightarrow Q_{n_{j}}\right) \leq r_{j, i} \text { for all } 1 \leq j \leq k, \quad 1 \leq i \leq n \text {. }
$$

Proposition 3.4 (Fulton, [F1, Prop. 8.1]). Assume that $\mathbf{r}$ is permissible and let $w$ be the corresponding permutation. Then $D_{\mathbf{r}}$ has codimension $\ell(w)$ in $F\left(n_{1}, \ldots, n_{k}\right)$ and its class in the Chow ring is given by a universal polynomial in the Chern classes of the bundles $Q_{n_{i}}$.

With this preparation we can study now the locus $\tilde{\Omega}_{w}^{\bar{e}}(t)$ defined in Lemma 3.1.

Construction 3.5. Let $k=\operatorname{card}\left\{i-e_{i} \mid i=1, \ldots, n-1\right\}$. Notice that $1-e_{1} \leq$ $\cdots \leq n-1-e_{n-1}$, because of the condition (2.1.2) on the multiindex $\bar{e}$. Set $e_{0}=e_{n}=0$ and define a partition of $[0, n]$ as follows:

$$
i_{0}=0, i_{j}=\min \left\{i \mid i-e_{i} \geq i_{j-1}-e_{i_{j-1}}+1\right\} \text {, for } 1 \leq j \leq k, i_{k+1}=n .
$$

Let $n_{j}=i_{j}-e_{i_{j}}$, for $j=0,1, \ldots, k$, and let $F\left(n_{1}, \ldots, n_{k}\right)$ be the corresponding partial flag variety.

Over $\mathcal{U}_{\bar{e}}(t)=\pi^{-1}\left(\{t\} \times \mathcal{H} \mathcal{Q}_{\bar{d}-\bar{e}}\right)$ all the maps

$$
V \otimes \mathcal{O} \rightarrow K_{n-1}^{*}, K_{n-1}^{*} \rightarrow K_{n-2}^{*}, \ldots, K_{2}^{*} \rightarrow K_{1}^{*}
$$

are surjective. By the definition of $i_{1}, \ldots, i_{k}$, on each of the intervals

$$
\left[1, i_{1}-1\right],\left[i_{1}, i_{2}-1\right], \ldots,\left[i_{k-1}, i_{k}-1\right],\left[i_{k}, n-1\right]
$$

$i-e_{i}$ is constant, equal respectively to $0, n_{1}, \ldots, n_{k}$, and the corresponding bundles $K_{i}^{*}$ are all isomorphic. Therefore the rank conditions defining $\tilde{\Omega}_{w}^{\bar{e}}(t)$ inside $\mathcal{U}_{\bar{e}}(t)$ are

$$
\operatorname{rank}\left(V_{p} \otimes \mathcal{O} \rightarrow K_{q}^{*}\right) \leq r_{w}(q, p), \quad 1 \leq p \leq n, \quad q \in\left\{i_{1}, \ldots, i_{k}\right\} .
$$

Define recursively $\mathbf{r}=\left(r_{j, p}\right)_{1 \leq p \leq n, 1 \leq j \leq k}$ as follows:

$$
\begin{gathered}
r_{1, p}=\min \left\{r_{w}\left(i_{1}, p\right), n_{1}\right\}, 1 \leq p \leq n, \\
r_{j, p}=\min \left\{r_{w}\left(i_{j}, p\right), r_{j-1, p}+n_{j}-n_{j-1}\right\}, 1 \leq p \leq n, 2 \leq j \leq k .
\end{gathered}
$$

Claim. The conditions

$$
\operatorname{rank}\left(V_{p} \otimes \mathcal{O} \rightarrow K_{i_{j}}^{*}\right) \leq r_{j, p}, 1 \leq p \leq n, 1 \leq j \leq k
$$

define the same degeneracy locus $\tilde{\Omega}_{w}^{\bar{e}}(t)$ in $\mathcal{U}_{\bar{e}}(t)$. 
Proof. For each $m=1, \ldots, k$, define $A_{m}$ to be the degeneracy locus determined by

$$
\operatorname{rank}\left(V_{p} \otimes \mathcal{O} \rightarrow K_{i_{j}}^{*}\right) \leq r_{w}\left(i_{j}, p\right), 1 \leq p \leq n, 1 \leq j \leq m,
$$

and $B_{m}$ to be the locus determined by the same conditions, but with $r_{w}\left(i_{j}, p\right)$ replaced by $r_{j, p}$. We prove by induction on $m$ that $A_{m}=B_{m}$ as schemes.

For $m=1$, there is nothing to prove if $r_{w}\left(i_{1}, p\right) \leq n_{1}$, by the definition of $r_{1, p}$. On the other hand, if $r_{w}\left(i_{1}, p\right)>n_{1}$, then $A_{m}=B_{m}=\mathcal{U}_{\bar{e}}(t)$, so we are done in this case too.

Assume now that $A_{m-1}=B_{m-1}$. To finish the proof we show that

$$
\operatorname{rank}\left(V_{p} \otimes \mathcal{O} \rightarrow K_{i_{m}}^{*}\right) \leq r_{w}\left(i_{m}, p\right)
$$

and

$$
\operatorname{rank}\left(V_{p} \otimes \mathcal{O} \rightarrow K_{i_{m}}^{*}\right) \leq r_{m, p}
$$

give in fact the same degeneracy condition at points in $A_{m-1}$. This is obvious if $r_{w}\left(i_{m}, p\right) \leq r_{m-1, p}+n_{m}-n_{m-1}$. Hence we may assume

$$
r_{m, p}=r_{m-1, p}+n_{m}-n_{m-1}<r_{w}\left(i_{m}, p\right) .
$$

Suppose now that

$$
\operatorname{rank}\left(V_{p} \otimes \mathcal{O} \rightarrow K_{i_{m}}^{*}\right) \geq r_{m-1, p}+n_{m}-n_{m-1}+1
$$

at some point in $A_{m-1}$. Since $V_{p} \otimes \mathcal{O} \rightarrow K_{i_{m-1}}^{*}$ factors through $K_{i_{m}}^{*}$ and $K_{i_{m}}^{*} \rightarrow$ $K_{i_{m-1}}^{*}$ is surjective, we have

$$
\begin{aligned}
\operatorname{rank}\left(V_{p} \otimes \mathcal{O} \rightarrow K_{i_{m-1}}^{*}\right) & \geq \operatorname{rank}\left(V_{p} \otimes \mathcal{O} \rightarrow K_{i_{m}}^{*}\right)-\operatorname{rank}\left(\operatorname{ker}\left(K_{i_{m}}^{*} \rightarrow K_{i_{m-1}}^{*}\right)\right) \\
& =\operatorname{rank}\left(V_{p} \otimes \mathcal{O} \rightarrow K_{i_{m}}^{*}\right)-\left(n_{m}-n_{m-1}\right) \\
& \geq r_{m-1, p}+1,
\end{aligned}
$$

contradicting the assumption $A_{m-1}=B_{m-1}$. We conclude that if $r_{m, p}<r_{w}\left(i_{m}, p\right)$, then $A_{m}=B_{m}=A_{m-1}=B_{m-1}$. This finishes the induction step and the claim is proved.

Restricting the bundles to $\mathcal{U}_{\bar{e}}(t)$ defines an "evaluation at $t$ " morphism

$$
\Phi_{\bar{e}}(t): \mathcal{U}_{\bar{e}}(t) \longrightarrow F\left(n_{1}, \ldots, n_{k}\right),
$$

and $\tilde{\Omega}_{w}^{\bar{e}}(t)$ is the preimage under $\Phi_{\bar{e}}(t)$ of the degeneracy locus $D_{\mathbf{r}} \subset F\left(n_{1}, \ldots, n_{k}\right)$.

Lemma 3.6. $\left(r_{j, p}\right)$ form a permissible collection of rank numbers.

Proof. We construct a permutation $\tilde{w}^{\bar{e}} \in S_{n}$ as follows:

For each $j=0,1, \ldots, k+1$, define sets $W_{j}(w)$ by

$$
W_{0}(w)=\emptyset, W_{j}(w)=\left\{w(1), \ldots, w\left(i_{j}\right)\right\} .
$$

Also we define sets $Z_{j}(w)$, and ordered sets $\tilde{Z}_{j}(w)$, such that

(a) $\operatorname{card} Z_{j}(w)=i_{j}-n_{j-1}$,

(b) $\operatorname{card} \tilde{Z}_{j}(w)=n_{j}-n_{j-1}$,

(c) $\tilde{Z}_{j}(w) \cap \tilde{Z}_{j^{\prime}}(w)=\emptyset$ if $j \neq j^{\prime}$,

(d) $\bigcup_{j=1}^{k+1} \tilde{Z}_{j}(w)=[1, n]$,

by the following recursive procedure: 
Let $Z_{0}(w)=\tilde{Z}_{0}(w)=\emptyset$. If $\tilde{Z}_{i}(w)$ has been already defined for $i=0,1, \ldots, j-1$, let

$$
Z_{j}(w)=W_{j}(w) \backslash\left(\bigcup_{i=1}^{j-1} \tilde{Z}_{i}(w)\right) .
$$

Arrange $Z_{j}(w)$ in increasing order:

$$
Z_{j}(w)=\left\{z_{j, 1}<\cdots<z_{j, i_{j}-n_{j-1}}\right\} .
$$

Let

$$
\tilde{z}_{n_{j-1}+1}:=z_{j, 1}, \tilde{z}_{n_{j-1}+2}:=z_{j, 2}, \ldots, \tilde{z}_{n_{j}}:=z_{j, n_{j}-n_{j-1}},
$$

and

$$
\tilde{Z}_{j}(w):=\left\{\tilde{z}_{n_{j-1}+1}<\tilde{z}_{n_{j-1}+2}<\cdots<\tilde{z}_{n_{j}}\right\} .
$$

Note that it may happen that $\tilde{z}_{n_{j-1}}>\tilde{z}_{n_{j-1}+1}$ !

Now define $\tilde{w}^{\bar{e}}(q)=\tilde{z}_{q}$, for all $1 \leq q \leq n$. By definition, if $(q, p) \in \mathcal{E} s s\left(\tilde{w}^{\bar{e}}\right)$, then $\tilde{w}^{\bar{e}}(q)>\tilde{w}^{\bar{e}}(q+1)$, and so we have necessarily $q \in\left\{n_{1}, \ldots, n_{k}\right\}$. Furthermore, one checks easily that $r_{j, p}=r_{\tilde{w}^{e}}\left(n_{j}, p\right)$, for all $1 \leq p \leq n, 1 \leq j \leq k$. Hence $\tilde{w}^{\bar{e}}$ satisfies the conditions in Definition 3.3 and the Lemma is proved.

Example 3.7. Let $n=10, \bar{e}=(1,2,3,4,5,6,2,3,2)$. We want to compute $\tilde{w}_{0}^{\bar{e}}$ and $\tilde{w}_{1}^{\bar{e}}$ for the permutations

$$
w_{0}=\left(\begin{array}{cccccccccc}
1 & 2 & 3 & 4 & 5 & 6 & 7 & 8 & 9 & 10 \\
10 & 9 & 8 & 7 & 6 & 5 & 4 & 3 & 2 & 1
\end{array}\right)
$$

and

$$
w_{1}=\left(\begin{array}{cccccccccc}
1 & 2 & 3 & 4 & 5 & 6 & 7 & 8 & 9 & 10 \\
4 & 5 & 3 & 7 & 1 & 9 & 8 & 6 & 10 & 2
\end{array}\right) .
$$

We have $k=2, i_{0}=0, i_{1}=7, i_{2}=9, i_{3}=10, n_{0}=0, n_{1}=5, n_{2}=7, n_{3}=10$, and

$$
\begin{gathered}
Z_{1}\left(w_{0}\right)=\{10,9,8,7,6,5,4\} ; \quad \tilde{Z}_{1}\left(w_{0}\right)=\{4,5,6,7,8\}, \\
Z_{2}\left(w_{0}\right)=\{9,10,3,2\} ; \quad \tilde{Z}_{2}\left(w_{0}\right)=\{2,3\}, \\
Z_{3}\left(w_{0}\right)=\{9,10,1\} ; \quad \tilde{Z}_{3}\left(w_{0}\right)=\{1,9,10\},
\end{gathered}
$$

and therefore

$$
\tilde{w}_{0}^{\bar{e}}=\left(\begin{array}{llllllllll}
1 & 2 & 3 & 4 & 5 & 6 & 7 & 8 & 9 & 10 \\
4 & 5 & 6 & 7 & 8 & 2 & 3 & 1 & 9 & 10
\end{array}\right) .
$$

Similarly,

$$
\begin{gathered}
Z_{1}\left(w_{1}\right)=\{4,5,3,7,1,9,8\} ; \quad \tilde{Z}_{1}\left(w_{1}\right)=\{1,3,4,5,7\}, \\
Z_{2}\left(w_{1}\right)=\{8,9,6,10\} ; \quad \tilde{Z}_{2}\left(w_{1}\right)=\{6,8\}, \\
Z_{3}\left(w_{1}\right)=\{9,10,2\} ; \quad \tilde{Z}_{3}\left(w_{1}\right)=\{2,9,10\},
\end{gathered}
$$

and

$$
\tilde{w}_{1}^{\bar{e}}=\left(\begin{array}{cccccccccc}
1 & 2 & 3 & 4 & 5 & 6 & 7 & 8 & 9 & 10 \\
1 & 3 & 4 & 5 & 7 & 6 & 8 & 2 & 9 & 10
\end{array}\right)
$$


Note that since the construction in Lemma 3.6 involves arranging each of the sets $Z_{1}, \ldots, Z_{k+1}$ in increasing order, the resulting permutation $\tilde{w}^{\bar{e}}$ will satisfy the inequality $\ell\left(\tilde{w}^{\bar{e}}\right) \leq \ell(w)$.

We now want to estimate $\ell(w)-\ell\left(\tilde{w}^{\bar{e}}\right)$, i.e. the number of inversions in $w$ that are killed by the procedure described above.

Lemma 3.8. $\ell(w)-\ell\left(\tilde{w}^{\bar{e}}\right) \leq \sum_{i=1}^{n-1} e_{i}$.

Proof. Denote as usual by $w_{0}$ the permutation of longest length

$$
w_{0}=\left(\begin{array}{ccccc}
1 & 2 & \ldots & n-1 & n \\
n & n-1 & \ldots & 2 & 1
\end{array}\right) .
$$

It is clear from the construction that

$$
\ell(w)-\ell\left(\tilde{w}^{\bar{e}}\right) \leq \ell\left(w_{0}\right)-\ell\left(\tilde{w}_{0}^{\bar{e}}\right),
$$

for every $w \in S_{n}$, therefore it suffices to prove the Lemma for $w_{0}$.

We will count the number of inversions killed in each step of the procedure described in Lemma 3.6. First

$$
Z_{1}\left(w_{0}\right)=\left\{n, n-1, \ldots, n-i_{1}+1\right\},
$$

hence, when ordering it increasingly, we destroy $\left(\begin{array}{c}i_{1} \\ 2\end{array}\right)$ inversions. Next we have

$$
Z_{2}\left(w_{0}\right)=\left\{n-i_{1}+1+n_{1}, \ldots, n-1, n, n-i_{1}, n-i_{1}-1, \ldots, n-i_{2}+1\right\},
$$

and when ordering it we destroy $\left(\begin{array}{c}i_{2}-i_{1} \\ 2\end{array}\right)+e_{i_{1}}\left(i_{2}-i_{1}\right)$ more inversions.

In general a similar counting shows that when ordering $Z_{j}\left(w_{0}\right)$ we destroy $\left(\begin{array}{c}i_{j}-i_{j-1} \\ 2\end{array}\right)+e_{i_{j-1}}\left(i_{j}-i_{j-1}\right)$ inversions for each $j=1, \ldots, k+1$. Hence

$$
\ell\left(w_{0}\right)-\ell\left(\tilde{w}_{0}^{\bar{e}}\right)=\sum_{j=1}^{k+1}\left(\left(\begin{array}{c}
i_{j}-i_{j-1} \\
2
\end{array}\right)+e_{i_{j-1}}\left(i_{j}-i_{j-1}\right)\right) .
$$

On the other hand, since $i-e_{i}$ is constant on $\left[i_{j-1}, i_{j}-1\right]$, for each $j=1, \ldots, k+1$, we have

$$
\begin{aligned}
\sum_{i=i_{j-1}}^{i_{j}-1} e_{i} & =e_{i_{j-1}}+\left(e_{i_{j-1}}+1\right)+\cdots+\left(e_{i_{j-1}}+\left(i_{j}-i_{j-1}-1\right)\right) \\
& =\left(\begin{array}{c}
i_{j}-i_{j-1} \\
2
\end{array}\right)+e_{i_{j-1}}\left(i_{j}-i_{j-1}\right) .
\end{aligned}
$$

If we add up the identities (3.8.2) for $j=1, \ldots, k+1$ and compare with (3.8.1), we get

$$
\ell\left(w_{0}\right)-\ell\left(\tilde{w}_{0}^{\bar{e}}\right)=\sum_{i=1}^{n-1} e_{i}
$$

We will need one more auxiliary result. 
Lemma 3.9. Let $\bar{e}=\left(e_{1}, \ldots, e_{n-1}\right)$ be a multiindex satisfying the conditions (2.1.1) and (2.1.2) of Lemma 2.1. Assume in addition that $\sum_{i=1}^{n-1} e_{i} \geq 1$. Then we have the following:

(i) $\sum_{i=1}^{n-1} e_{i}\left(e_{i}-e_{i-1}\right) \geq 1$.

(ii) We have equality in (i) iff there exist integers $1 \leq h \leq l \leq n-1$ such that $e_{i}=0$, for $i \in[1, h-1] \cup[l+1, n-1]$ and $e_{i}=1$, for $i \in[h, l]$, i.e., $\bar{e}$ consists of a (possibly empty) string of zeroes, followed by a string of 1's, followed by a (possibly empty) string of zeroes. We denote such a multiindex by $\bar{e}_{h l}$.

(iii) Let $\bar{e}=\bar{e}_{h l}$ be a multiindex as in (ii), let $w \in S_{n}$ be any permutation and let $\tilde{w}^{\bar{e}}$ be the permutation constructed in Lemma 3.6. Then

$$
\ell(w)-\ell\left(\tilde{w}^{\bar{e}}\right)=\sum_{i=1}^{n-1} e_{i}=l-h+1
$$

iff $w(h)>\max \{w(h+1), \ldots, w(l), w(l+1)\}$, and in this case $\tilde{w}^{\bar{e}}$ is the permutation $\left(\begin{array}{cccccccccc}1 & \ldots & h-1 & h & \ldots & l & l+1 & l+2 & \ldots & n \\ w(1) & \ldots & w(h-1) & w(h+1) & \ldots & w(l+1) & w(h) & w(l+2) & \ldots & w(n)\end{array}\right)$.

Proof. (i) and (ii) follow from the identity

$$
\sum_{i=1}^{n-1} e_{i}\left(e_{i}-e_{i-1}\right)=\frac{1}{2}\left[e_{1}^{2}+\left(e_{2}-e_{1}\right)^{2}+\cdots+\left(e_{n-1}-e_{n-2}\right)^{2}+e_{n-1}^{2}\right],
$$

while (iii) is an easy consequence of the construction of $\tilde{w}^{\bar{e}}$ in Lemma 3.6 .

\section{Gromov-Witten InVARIANts AND THE QUANTUM MULTiPliCATion MAP}

To define the quantum multiplication for cohomology classes on $F$ we will need the Gromov-Witten invariants associated to Schubert varieties. Following [Be2], we will define these invariants as certain intersection numbers on the hyperquot schemes. In order to check that this gives the correct invariants, a "general position" result is needed.

Theorem 4.1. (i) Let $Y$ be a fixed subvariety of $H_{\bar{d}}$. For any $w \in S$, a corresponding general translate of $\Omega_{w} \subset F$, and $t \in \mathbb{P}^{1}$, the intersection $Y \cap \Omega_{w}(t)$ is either empty or has pure codimension $\ell(w)$ in $Y$. In particular, for any permutations $w_{1}, \ldots, w_{N} \in S_{n}$, points $t_{1}, \ldots, t_{N} \in \mathbb{P}^{1}$, and general translates of $\Omega_{w_{i}} \subset F$, the intersection $\bigcap_{i=1}^{N} \Omega_{w_{i}}\left(t_{i}\right)$ is either empty or has pure codimension $\sum \ell\left(w_{i}\right)$ in $H_{\bar{d}}$.

(ii) Moreover, if $t_{1}, \ldots, t_{N}$ are distinct, then for general translates of the $\Omega_{w_{i}}$ the intersection $\bigcap_{i=1}^{N} \bar{\Omega}_{w_{i}}\left(t_{i}\right)$ is either empty or has pure codimension $\sum_{i=1}^{N} \ell\left(w_{i}\right)$ in $\mathcal{H} \mathcal{Q}_{\bar{d}}$ and is the Zariski closure of $\bigcap_{i=1}^{N} \Omega_{w_{i}}\left(t_{i}\right)$.

Proof. (i) This follows from a Bertini-type theorem of Kleiman ([Kl]), since $F$ is a homogeneous space. A very short proof can be found also in [Be2, Lemma 2.2].

(ii) The proof is by induction on $\left(d_{1}, \ldots, d_{n-1}\right)$ and is similar to that in $[\mathrm{Be} 2$, Lemma $2.2 \mathrm{~A}]$.

If $d_{1}=\cdots=d_{n-1}=0$, then $\mathcal{H} \mathcal{Q}_{\bar{d}}=H_{\bar{d}}=F$ and (ii) reduces to the statement that general translates of Schubert varieties on $F$ intersect transversely. Assume that the statement is true for all $\bar{f} \in \mathbb{N}^{n-1}$ such that $f_{i} \leq d_{i}, 1 \leq i \leq n-1$, and $f_{j}<d_{j}$ for some $1 \leq j \leq n-1$. 
Let $c=\sum_{i=1}^{N} \ell\left(w_{i}\right)$ and denote for each (nonzero) multiindex $\bar{e}=\left(e_{1}, \ldots, e_{n-1}\right)$, satisfying the conditions (2.1.1) and (2.1.2) of Lemma 2.1,

$$
T_{\bar{e}}:=h_{\bar{e}}\left(\mathcal{U}_{\bar{e}}\right) \cap\left(\bigcap_{i=1}^{N} \bar{\Omega}_{w_{i}}\left(t_{i}\right)\right) .
$$

By $(i)$ and Theorem $2.3(i i)$, we only need to prove the inequality

$$
\operatorname{codim}_{\mathcal{H} \mathcal{Q}_{\bar{d}}} T_{\bar{e}}>c
$$

for every $\bar{e}$. Since $h_{\bar{e}}$ is birational onto its image, this will follow if we show that

$$
\begin{aligned}
\operatorname{codim}_{\mathcal{U}_{\bar{e}}}\left(\bigcap_{i=1}^{N} h_{\bar{e}}^{-1}\left(\bar{\Omega}_{w_{i}}\left(t_{i}\right)\right)\right) & >c-\left(\operatorname{dim} \mathcal{H} \mathcal{Q}_{\bar{d}}-\operatorname{dim} \mathcal{U}_{\bar{e}}\right) \\
& =c+1-\sum_{i=1}^{n-1} e_{i}\left(1+e_{i}-e_{i-1}\right) .
\end{aligned}
$$

By Lemma 3.1

$$
\bigcap_{i=1}^{N} h_{\bar{e}}^{-1}\left(\bar{\Omega}_{w_{i}}\left(t_{i}\right)\right)=\bigcap_{i=1}^{N}\left(\pi^{-1}\left(\mathbb{P}^{1} \times \bar{\Omega}_{w_{i}}\left(t_{i}\right)\right) \cup \tilde{\Omega}_{w_{i}}^{\bar{e}}\left(t_{i}\right)\right)
$$

Since each $\tilde{\Omega}_{w_{i}}^{\bar{e}}\left(t_{i}\right)$ is supported on $\pi^{-1}\left(\left\{t_{i}\right\} \times \mathcal{H} \mathcal{Q}_{\bar{d}-\bar{e}}\right)$ and the points $t_{1}, \ldots, t_{N}$ are distinct, the only intersections that may contribute are of the type

$$
\bigcap_{i=1}^{N}\left(\pi^{-1}\left(\mathbb{P}^{1} \times \bar{\Omega}_{w_{i}}\left(t_{i}\right)\right)\right)
$$

and (after possibly renumbering the points)

$$
\bigcap_{i=1}^{N-1}\left(\pi^{-1}\left(\mathbb{P}^{1} \times \bar{\Omega}_{w_{i}}\left(t_{i}\right)\right)\right) \cap \tilde{\Omega}_{w_{N}}^{\bar{e}}\left(t_{N}\right) .
$$

For intersections of type $(*)$ the codimension estimate (4.1.1) follows easily from the induction hypothesis, as $\pi$ is a smooth map.

It remains to deal with the intersections of type $\left({ }^{* *}\right)$. Notice first that any such intersection is supported on $\pi^{-1}\left(\left\{t_{N}\right\} \times \mathcal{H} \mathcal{Q}_{\bar{d}-\bar{e}}\right)$. If we let

$$
W:=\bigcap_{i=1}^{N-1}\left(\pi^{-1}\left(\left\{t_{N}\right\} \times \bar{\Omega}_{w_{i}}\left(t_{i}\right)\right)\right),
$$

then $W$ has codimension $c-\ell\left(w_{N}\right)$ in $\mathcal{U}_{\bar{e}}\left(t_{N}\right)$, and by the Construction 3.5, Lemma 3.6, Proposition 3.4, and Kleiman's Theorem again (partial flag varieties are homogeneous!), we get that $W \cap \tilde{\Omega}_{w_{N}}^{\bar{e}}\left(t_{N}\right)$ has codimension $\ell\left(\tilde{w}_{N}^{\bar{e}}\right)$ in $W$. Therefore $\left(^{* *}\right)$ has codimension $c-\ell\left(w_{N}\right)+\ell\left(\tilde{w}_{N}^{\bar{e}}\right)+1$ in $\mathcal{U}_{\bar{e}}$. By Lemma 3.8

$$
c-\ell\left(w_{N}\right)+\ell\left(\tilde{w}_{N}^{\bar{e}}\right)+1 \geq c+1-\sum_{i=1}^{n-1} e_{i},
$$

and by Lemma $3.9(i)$

$$
\sum_{i=1}^{n-1} e_{i}\left(1+e_{i}-e_{i-1}\right)=\sum_{i=1}^{n-1} e_{i}\left(e_{i}-e_{i-1}\right)+\sum_{i=1}^{n-1} e_{i} \geq 1+\sum_{i=1}^{n-1} e_{i} .
$$

The required inequality follows by combining (4.1.2) and (4.1.3). 
With exactly the same arguments as in [Be2, Cor. 2.3 and Cor. 2.4] we get

Corollary 4.2. The class of $\bar{\Omega}_{w}(t)$ in $C H^{\ell(w)}\left(\mathcal{H} \mathcal{Q}_{\bar{d}}\right)$ is independent of $t \in \mathbb{P}^{1}$ and the flag $V_{\bullet} \subset V$.

Let $\mathcal{D}=\frac{n(n-1)}{2}+2 \sum_{i=1}^{n-1} d_{i}=\operatorname{dim}\left(\mathcal{H} \mathcal{Q}_{\bar{d}}\right)$.

Corollary 4.3. If $\sum_{i=1}^{N} \ell\left(w_{i}\right)=\mathcal{D}$ and $t_{1}, \ldots, t_{N}$ are distinct, then the number of points in $\bigcap_{i=1}^{N} \Omega_{w_{i}}\left(t_{i}\right)$ can be computed as the degree of the product $\bar{\Omega}_{w_{1}}\left(t_{1}\right)$. $\cdots \bar{\Omega}_{w_{N}}\left(t_{N}\right)$ in the Chow ring $C H^{*}\left(\mathcal{H} \mathcal{Q}_{\bar{d}}\right)$, hence it is independent of $t_{i}$ and the (general) translates of $\Omega_{w_{i}}$.

The corollaries imply that, for general translates of $\Omega_{w_{1}}, \ldots, \Omega_{w_{N}}$, we have a well-defined intersection number

$$
\left\langle\Omega_{w_{1}}, \ldots, \Omega_{w_{N}}\right\rangle_{\bar{d}}:= \begin{cases}\text { number of points in } \bigcap_{i=1}^{N} \Omega_{w_{i}}\left(t_{i}\right), & \text { if } \sum_{i=1}^{N} \ell\left(w_{i}\right)=\mathcal{D}, \\ 0, & \text { otherwise. }\end{cases}
$$

This is the Gromov-Witten invariant associated to the classes $\Omega_{w_{1}}, \ldots, \Omega_{w_{N}}$.

Definition 4.4. The quantum multiplication map is the linear map

$$
m_{q}: \operatorname{Sym}\left(H^{*}(F ; \mathbb{Z})\left[q_{1}, \ldots, q_{n-1}\right]\right) \rightarrow H^{*}(F ; \mathbb{Z})\left[q_{1}, \ldots, q_{n-1}\right]
$$

given by

$$
m_{q}\left(\prod_{i=1}^{N} \Omega_{w_{i}} \bar{q}^{\bar{m}_{i}}\right)=\bar{q}^{\sum \bar{m}_{i}} \sum_{\bar{d} \in \mathbb{N}^{n-1}} \bar{q}^{\bar{d}}\left(\sum_{w \in S_{n}}\left\langle\Omega_{w}, \Omega_{w_{1}}, \ldots, \Omega_{w_{N}}\right\rangle_{\bar{d}} \Omega_{w_{0} \cdot w}\right) .
$$

Here $\bar{q}^{\bar{m}}$ denotes as usual the monomial $\prod_{i=1}^{n-1} q_{i}^{m_{i}}$.

The following is an immediate consequence of Theorem 4.1 (see e.g. [Be2]).

Lemma 4.5. The map $m_{q}$ induces the identity on $\operatorname{Sym}^{1}\left(H^{*}(F ; \mathbb{Z})\left[q_{1}, \ldots, q_{n-1}\right]\right)$. Equivalently, if $\bar{d} \neq(0, \ldots, 0)$, every Gromov-Witten invariant $\left\langle\Omega_{w_{1}}, \Omega_{w_{2}}\right\rangle_{\bar{d}}$ involving only two Schubert classes vanishes.

The map $m_{q}$ defined above induces a pairing

$$
\operatorname{Sym}^{2}\left(H^{*}(F ; \mathbb{Z})\left[q_{1}, \ldots, q_{n-1}\right]\right) \longrightarrow H^{*}(F ; \mathbb{Z})\left[q_{1}, \ldots, q_{n-1}\right]
$$

and there are by now several successful approaches to prove that this gives a new ring structure on $H^{*}(F ; \mathbb{Z})\left[q_{1}, \ldots, q_{n-1}\right]$ ([BM], [KM], [LT1], [LT2], [RT]). We explain below why our seemingly ad-hoc definition of quantum multiplication agrees with the general one.

In general Gromov-Witten invariants are defined algebro-geometrically as intersection numbers on Kontsevich's space of stable pointed maps, which exists for every smooth variety (see $[\mathrm{KM}]$ ). Since $F$ is homogeneous, the three-point invariant (in the notation of $[\mathrm{KM}])$

$$
\left\langle I_{0,3, \bar{d}}^{F}\right\rangle\left(\Omega_{w_{1}} \otimes \Omega_{w_{2}} \otimes \Omega_{w_{3}}\right)
$$

gives the number of maps from $\mathbb{P}^{1}$ to $F$ of multidegree $\bar{d}$, mapping three given points (e.g. 0,1 and $\infty$ ) to $\Omega_{w_{1}}, \Omega_{w_{2}}$ and $\Omega_{w_{3}}$ respectively (see [FP, Lemma 14] for a proof of this). Hence $\left\langle I_{0,3, \bar{d}}^{F}\right\rangle\left(\Omega_{w_{1}} \otimes \Omega_{w_{2}} \otimes \Omega_{w_{3}}\right)$ coincides with the three-point invariant $\left\langle\Omega_{w_{1}}, \Omega_{w_{2}}, \Omega_{w_{3}}\right\rangle_{\bar{d}}$ of $F$ as defined above. Since the quantum multiplication can be given only in terms of three-point invariants, it follows that we have 
defined in Definition 4.4 the correct map $m_{q}$. Note however that the invariants $\left\langle\Omega_{w_{1}}, \ldots, \Omega_{w_{N}}\right\rangle_{\bar{d}}$ and $\left\langle I_{0, N, \bar{d}}^{F}\right\rangle\left(\Omega_{w_{1}} \otimes \cdots \otimes \Omega_{w_{N}}\right)$ are not equal when $N \neq 3$ (they are solutions to different enumerative problems).

Definition 4.6. $H^{*}(F ; \mathbb{Z})\left[q_{1}, \ldots, q_{n-1}\right]$, together with the multiplication $m_{q}$, is called the (small) quantum cohomology ring of $F$.

Recall from Section 0 the presentation

$$
H^{*}(F ; \mathbb{Z}) \cong \mathbb{Z}\left[x_{1}, x_{2}, \ldots, x_{n}\right] /\left(R_{1}(n), \ldots, R_{n}(n)\right) .
$$

There is an induced map

$$
m_{q}: \mathbb{Z}\left[x_{1}, x_{2}, \ldots, x_{n}, q_{1}, q_{2}, \ldots, q_{n-1}\right] \rightarrow H^{*}(F ; \mathbb{Z})\left[q_{1}, \ldots, q_{n-1}\right],
$$

which is surjective (see [ST, Lemma 2.1] for an easy argument by induction on degree). Let $I_{q}$ be the kernel of this map. Then $I_{q}$ is an ideal with respect to the usual polynomial ring structure on $\mathbb{Z}\left[x_{1}, \ldots, x_{n}, q_{1}, \ldots, q_{n-1}\right]$. Thus $m_{q}$ defines a $\mathbb{Z}\left[q_{1}, \ldots, q_{n-1}\right]$-algebra structure on $H^{*}(F ; \mathbb{Z})\left[q_{1}, \ldots, q_{n-1}\right]$ which is isomorphic to

$$
\mathbb{Z}\left[x_{1}, x_{2}, \ldots, x_{n}, q_{1}, q_{2}, \ldots, q_{n-1}\right] / I_{q} .
$$

\section{The quantum Cohomology RING of $F$}

Since the generators $x_{i}$ of the usual cohomology ring can be written linearly in terms of the "basic" Schubert classes $\Omega_{s_{i}}$, to determine the ring structure on $H^{*}(F ; \mathbb{Z})$ one should know how to calculate a product of the type $\Omega_{s_{i}} \cdot \Omega_{w}$.

Let $M_{i, w}$ denote the class given by the product $\Omega_{s_{i}} \cdot \Omega_{w}$ in the cohomology of $F$. Monk's formula (see [Mo]) expresses this class in terms of the additive basis given by Schubert varieties:

$$
M_{i, w}=\sum_{t_{j k}} \Omega_{w \cdot t_{j k}},
$$

summed over all transpositions $t_{j k}$ of integers $j \leq i<k$ such that $\ell\left(w \cdot t_{j k}\right)=$ $\ell(w)+1$.

We will prove a similar formula for the quantum multiplication of $\Omega_{s_{i}}$ and $\Omega_{w}$.

Since the quantum multiplication is defined by specifying the Gromov-Witten invariants as structure constants, we need to figure out $\left\langle\Omega_{s_{i}}, \Omega_{w}, \Omega_{w^{\prime}}\right\rangle_{\bar{d}}$ for all the permutations $w^{\prime} \in S_{n}$ such that $\ell(w)+\ell\left(w^{\prime}\right)+1=n(n-1) / 2+2 \sum_{i=1}^{n-1} d_{i}$.

For the rest of this section we will always work with general translates of the Schubert classes on $F$. By Theorem 4.1 and its two corollaries,

$$
\left\langle\Omega_{s_{i}}, \Omega_{w}, \Omega_{w^{\prime}}\right\rangle_{\bar{d}}=\operatorname{card}\left(\bar{\Omega}_{s_{i}}(u) \cap \bar{\Omega}_{w}(v) \cap \bar{\Omega}_{w^{\prime}}(t)\right)=\operatorname{deg}\left(\bar{\Omega}_{s_{i}}(u) \cdot \bar{\Omega}_{w}(v) \cdot \bar{\Omega}_{w^{\prime}}(t)\right)
$$

where $u, v, t \in \mathbb{P}^{1}$ are distinct points and the product in the second line is computed in $C H^{*}\left(\mathcal{H} \mathcal{Q}_{\bar{d}}\right)$. Moreover, we know that the (transverse) intersection in the first line is contained in $H_{\bar{d}}$. Assume now that when we allow some of the points to coincide, the intersection remains transverse (see Lemma 5.1). Then, by Corollary 4.3 , its cardinality will still give

$$
\left\langle\Omega_{s_{i}}, \Omega_{w}, \Omega_{w^{\prime}}\right\rangle_{\bar{d}}
$$

however, some of the intersection points may lie now in the boundary. Let us analyze in more detail what happens if we let $u=v$ only and keep $t$ distinct. Recall the evaluation map

$$
e v: \mathbb{P}^{1} \times H_{\bar{d}} \rightarrow F, \quad e v(u, f)=f(u) .
$$


It is easy to see that this map is smooth. In particular, its restriction $e v_{u}$ to $\{u\} \times H_{\bar{d}}$ is also smooth. Let $Y$ denote the intersection $\Omega_{s_{i}} \cap \Omega_{w}$ inside $F$. By choosing suitable general translates, we may assume that $Y$ is irreducible, of codimension $\ell(w)+1$ in $F$, and the intersection

$$
\Omega_{s_{i}}(u) \cap \Omega_{w}(u) \cap \Omega_{w^{\prime}}(t)=e v_{u}^{-1}(Y) \cap \Omega_{w^{\prime}}(t)
$$

is either empty, or it consists of finitely many reduced points in $H_{\bar{d}}$. We claim that it is in fact empty. Indeed, we can interpret a point in the above intersection as a map $f: \mathbb{P}^{1} \rightarrow F$ such that $f(u) \in Y$ and $f(t) \in \Omega_{w^{\prime}}$. However, if such a map exists, then there is an entire 1-dimensional family of them, obtained by composing $f$ with the automorphisms of $\mathbb{P}^{1}$ which fix $u$ and $t$. (This is exactly the argument giving Lemma 4.5.)

We conclude therefore that the intersection of the closures

$$
\bar{\Omega}_{s_{i}}(u) \cap \bar{\Omega}_{w}(u) \cap \bar{\Omega}_{w^{\prime}}(t)
$$

is supported on the boundary of $\mathcal{H} \mathcal{Q}_{\bar{d}}$.

What is more remarkable is that the explicit description in Sections 2-4 allows us to calculate the contribution in the boundary as well. Assume that $\bar{d} \neq(0, \ldots, 0)$, and let $Z$ denote the intersection (5.1.1).

Lemma 5.1. (i) $Z$ is either empty or has pure codimension $\mathcal{D}=n(n-1) / 2+$ $2 \sum_{i=1}^{n-1} d_{i}$ in $\mathcal{H} \mathcal{Q}_{\bar{d}}$.

(ii) $Z$ is contained in $\bigcup_{\bar{e}_{h l}} h_{\bar{e}_{h l}}\left(\mathcal{U}_{\bar{e}_{h l}}(u)\right)$, the union over all the indices $\bar{e}_{h l}$ as in Lemma 3.9 (ii), with $h \leq i \leq l$. (recall that $\left.\mathcal{U}_{\bar{e}}(u)=\pi^{-1}\left(\{u\} \times \mathcal{H} \mathcal{Q}_{\bar{d}-\bar{e}}\right)\right)$.

(iii) If $Z \cap h_{\bar{e}_{h l}}\left(\mathcal{U}_{\bar{e}_{h l}}(u)\right)$ is nonempty, then $w$ satisfies the condition

$$
w(h)>\max \{w(h+1), \ldots, w(l), w(l+1)\}
$$

of Lemma 3.9 (iii). Moreover, in this case there is an equality (of sets)

$$
Z \cap h_{\bar{e}_{h l}}\left(\mathcal{U}_{\bar{e}_{h l}}(u)\right)=h_{\bar{e}_{h l}}\left(\tilde{\Omega}_{w}^{\bar{e}_{h l}}(u) \cap \pi^{-1}\left(\{u\} \times \bar{\Omega}_{w^{\prime}}(t)\right)\right) .
$$

Proof. (i) It suffices to show that

$$
h_{\bar{e}}\left(\mathcal{U}_{\bar{e}}\right) \cap \bar{\Omega}_{s_{i}}(u) \cap \bar{\Omega}_{w}(u) \cap \bar{\Omega}_{w^{\prime}}(t)
$$

has codimension at least $\mathcal{D}$ in $\mathcal{H} \mathcal{Q}_{\bar{d}}$, for every $\bar{e} \neq(0, \ldots, 0)$ satisfying the conditions (2.1.1)-(2.1.2). By repeating the reasoning in the proof of Theorem 4.1 (ii), we are reduced to proving that

$$
h_{\bar{e}}^{-1}\left(\bar{\Omega}_{s_{i}}(u)\right) \cap h_{\bar{e}}^{-1}\left(\bar{\Omega}_{w}(u)\right) \cap h_{\bar{e}}^{-1}\left(\bar{\Omega}_{w^{\prime}}(t)\right)
$$

has codimension at least

$$
\mathcal{D}+1-\sum_{i=1}^{n-1} e_{i}\left(1+e_{i}-e_{i-1}\right)
$$

in $\mathcal{U}_{\bar{e}}$. Using Lemma 3.1 , we can again treat this as a union of several types of intersections. This time however, due to the fact that not all the points in $\mathbb{P}^{1}$ are distinct, there are three types that may contribute. The first two of these are the types $(*)$ and $\left({ }^{*}\right)$ discussed in the proof of $4.1(i i)$, and we have already seen that their codimension in $\mathcal{U}_{\bar{e}}$ is greater than $\mathcal{D}+1-\sum_{i=1}^{n-1} e_{i}\left(1+e_{i}-e_{i-1}\right)$, so they are actually empty. The only (possibly) nonempty intersection is then

$$
W:=\tilde{\Omega}_{s_{i}}^{\bar{e}}(u) \cap \tilde{\Omega}_{w}^{\bar{e}}(u) \cap \pi^{-1}\left(\mathbb{P}^{1} \times \bar{\Omega}_{w^{\prime}}(t)\right),
$$


which is contained in $\mathcal{U}_{\bar{e}}(u)$. The same argument as in $4.1(i i)$ shows that

$$
\operatorname{codim}_{\mathcal{U}_{\bar{e}}} W \geq \mathcal{D}+1-\left(\ell\left(s_{i}\right)-\ell\left(\tilde{s}_{i}^{\bar{e}}\right)\right)-\left(\ell(w)-\ell\left(\tilde{w}^{\bar{e}}\right)\right) \geq \mathcal{D}-\sum_{i=1}^{n-1} e_{i},
$$

where the second inequality in (5.1.4) follows from the two estimates $\ell\left(s_{i}\right)-\ell\left(\tilde{s}_{i}^{\bar{e}}\right) \leq 1$ (which is obvious) and $\ell(w)-\ell\left(\tilde{w}^{\bar{e}}\right) \leq \sum_{i=1}^{n-1} e_{i}$ (Lemma 3.8). Using now Lemma 3.9 $(i)$, we obtain from (5.1.4) that the bound (5.1.3) holds as well for the codimension of $W$ in $\mathcal{U}_{\bar{e}}$.

The statements $(i i)$ and $($ iii) are immediate from Lemma $3.9(i i),(i i i)$. Indeed, if the intersection $Z \cap h_{\bar{e}}\left(\mathcal{U}_{\bar{e}}(u)\right)$ is nonempty, all the inequalities we have used in the proof of $(i)$ to estimate its codimension must in fact be equalities.

Corollary 5.2. $\left\langle\Omega_{s_{i}}, \Omega_{w}, \Omega_{w^{\prime}}\right\rangle_{\bar{d}}=\operatorname{length}(Z)$.

As a warm-up (and because this is the main step for the proof of Theorem 5.6), we will compute now the Gromov-Witten invariants and prove the quantum Monk formula for a special class of permutations.

For $m \leq n, k \leq m-1$, let $\alpha_{k, m} \in S_{n}$ be the cycle

$$
\left(\begin{array}{cccccccccc}
1 & 2 & \ldots & m-k & m-k+1 & \ldots & m-1 & m & m+1 \ldots n \\
1 & & 2 & \ldots & m-k & m-k+2 & \ldots & m & m-k+1 & m+1 \ldots n
\end{array}\right)
$$

Proposition 5.3. $m_{q}\left(\Omega_{s_{i}} \Omega_{\alpha_{k, m}}\right)=m_{q}\left(M_{i, \alpha_{k, m}}\right)+\delta_{i, m-1} q_{i} m_{q}\left(\Omega_{\alpha_{k, m} \cdot s_{i}}\right)$, where $\delta_{i, m-1}$ is the Kronecker symbol.

Proof. Let $\bar{d}$ be a multiindex and let $w$ be a permutation such that $\ell(w)=\mathcal{D}-$ $\ell\left(s_{i}\right)-\ell\left(\alpha_{k, m}\right)$. We want to evaluate $\left\langle\Omega_{s_{i}}, \Omega_{\alpha_{k, m}}, \Omega_{w}\right\rangle_{\bar{d}}$ by the procedure described above.

By Lemma $5.1($ ii $),($ iii $)$, there may be a nontrivial contribution in the boundary only if $i=m-1$ and it comes from

$$
h_{\bar{e}_{m-1}}\left(\tilde{\Omega}_{\alpha_{k, m}}^{\bar{e}_{m-1}}(u) \cap \pi^{-1}\left(\{u\} \times \bar{\Omega}_{w}(t)\right)\right) .
$$

Moreover, $\tilde{\alpha}_{k, m}^{\bar{e}_{m-1}}=\alpha_{k, m} \cdot s_{m-1}$, by Lemma 3.9 (iii). In particular, note that $(m-1, p)$ is not in $\mathcal{E} s s\left(\alpha_{k, m} \cdot s_{m-1}\right)$, for every $1 \leq p \leq n$. On the other hand, $\pi$ : $\mathcal{U}_{\bar{e}_{m-1}} \rightarrow \mathbb{P}^{1} \times \mathcal{H} \mathcal{Q}_{\bar{d}-\bar{e}_{m-1}}$ is an open immersion, whose image contains $\mathbb{P}^{1} \times H_{\bar{d}-\bar{e}_{m-1}}$ (see Remark 2.5). From these observations and the Construction 3.5, it follows that

$$
\tilde{\Omega}_{\alpha_{k, m}}^{\bar{e}_{m-1}}(u)=\bar{\Omega}_{\alpha_{k, m} \cdot s_{m-1}}(u) \cap \mathcal{U}_{\bar{e}_{m-1}} \subset\{u\} \times \mathcal{H} \mathcal{Q}_{\bar{d}-\bar{e}_{m-1}} .
$$

Therefore, by Theorem 4.1,

$$
\tilde{\Omega}_{\alpha_{k, m}}^{\bar{e}_{m-1}}(u) \cap \pi^{-1}\left(\{u\} \times \bar{\Omega}_{w}(t)\right)=\Omega_{\alpha_{k, m} \cdot s_{m-1}}(u) \cap \Omega_{w}(t) .
$$

By Lemma 4.5 , this intersection is empty, unless $\bar{d}=\bar{e}_{m-1}$ and $\Omega_{w}$ is the complementary Schubert variety for $\Omega_{\alpha_{k, m} \cdot s_{m-1}}$, in which case it is a single point. Moreover, in this case $\mathcal{U}_{\bar{e}_{m-1}} \cong \mathbb{P}^{1} \times F$ and $h_{\bar{e}_{m-1}}$ is an embedding. We conclude that $\left\langle\Omega_{s_{m-1}}, \Omega_{\alpha_{k, m}}, \Omega_{w}\right\rangle_{\bar{d}}=1$. The formula in the proposition is now immediate.

The following example, which was pointed out to us by W. Fulton, shows that in general the boundary contributions will come as well from the deeper strata $h_{\bar{e}_{h l}}\left(\mathcal{U}_{\bar{e}_{h l}}\right)$, with $h-l+1 \geq 2$. 
Example 5.4. Let $n=3$, and let $w_{0}=(321)$ be the permutation of longest length. We want to compute $m_{q}\left(\Omega_{s_{1}} \Omega_{w_{0}}\right)$. For dimension reasons, the only Gromov-Witten invariants that may be nonzero are

$$
\begin{gathered}
\left\langle\Omega_{s_{1}}, \Omega_{w_{0}}, \Omega_{s_{1}}\right\rangle_{(1,0)},\left\langle\Omega_{s_{1}}, \Omega_{w_{0}}, \Omega_{s_{1}}\right\rangle_{(0,1)},\left\langle\Omega_{s_{1}}, \Omega_{w_{0}}, \Omega_{s_{2}}\right\rangle_{(1,0)}, \\
\left\langle\Omega_{s_{1}}, \Omega_{w_{0}}, \Omega_{s_{2}}\right\rangle_{(0,1)},\left\langle\Omega_{s_{1}}, \Omega_{w_{0}}, \Omega_{w_{0}}\right\rangle_{(1,1)} .
\end{gathered}
$$

By the same reasoning as in Proposition 5.3, one computes easily

$$
\left\langle\Omega_{s_{1}}, \Omega_{w_{0}}, \Omega_{s_{1}}\right\rangle_{(0,1)}=\left\langle\Omega_{s_{1}}, \Omega_{w_{0}}, \Omega_{s_{2}}\right\rangle_{(1,0)}=\left\langle\Omega_{s_{1}}, \Omega_{w_{0}}, \Omega_{s_{2}}\right\rangle_{(0,1)}=0
$$

and

$$
\left\langle\Omega_{s_{1}}, \Omega_{w_{0}} \Omega_{s_{1}}\right\rangle_{(1,0)}=1 .
$$

To analyze $\left\langle\Omega_{s_{1}}, \Omega_{w_{0}}, \Omega_{w_{0}}\right\rangle_{(1,1)}$, recall that we have described completely in Example 2.6 the boundary of $\mathcal{H} \mathcal{Q}_{(1,1)}$. If $u, t \in \mathbb{P}^{1}$ are distinct points, the intersection

$$
\bar{\Omega}_{s_{1}}(u) \cap \bar{\Omega}_{w_{0}}(u) \cap \bar{\Omega}_{w_{0}}(t)
$$

is supported on $D_{1} \cup D_{2} \cup E$. Since $\tilde{s}_{1}^{(0,1)}=s_{1}$, there will be no contribution from $D_{2}$. On the other hand, $\tilde{s}_{1}^{(1,0)}=(123)$ and $\tilde{w}_{0}^{(1,0)}=(231)$, hence on $D_{1} \cong \mathbb{P}^{1} \times H_{(0,1)}$ the contribution is given by

$$
\{u\} \times \Omega_{\tilde{w}_{0}^{(1,0)}}(u) \cap \mathbb{P}^{1} \times \Omega_{w_{0}}(t)
$$

By Lemma 4.5, this last intersection is empty. We must still deal with $E$. We have

$$
\tilde{w}_{0}^{(1,1)}=s_{1}, \quad \tilde{s}_{1}^{(1,1)}=(123),
$$

hence $\tilde{\Omega}_{w_{0}}(u)$ is the locus inside the $\mathbb{P}^{1}$-bundle

$$
\pi^{-1}(\{u\} \times F) \rightarrow\{u\} \times F
$$

where $V_{1} \rightarrow K_{2}^{*}$ vanishes, and $\tilde{\Omega}_{s_{1}}(u)=\pi^{-1}(\{u\} \times F)$. The intersection

$$
\tilde{\Omega}_{w_{0}}(u) \cap \pi^{-1}\left(\{u\} \times \Omega_{w_{0}}(t)\right)
$$

is then obviously a single point (the first term is a section, while the second is a fibre). Hence $\left\langle\Omega_{s_{1}}, \Omega_{w_{0}}, \Omega_{w_{0}}\right\rangle_{(1,1)}=1$, and

$$
m_{q}\left(\Omega_{s_{1}} \Omega_{w_{0}}\right)=q_{1} m_{q}\left(\Omega_{(231)}\right)+q_{1} q_{2} m_{q}\left(\Omega_{(123)}\right) .
$$

A similar reasoning gives the general quantum Monk formula. As we mentioned in the introduction, this formula was obtained first (in a different way) in [FGP].

Theorem 5.5 (quantum Monk formula). For every $w \in S_{n}$ and every $1 \leq i \leq$ $n-1$,

$$
m_{q}\left(\Omega_{s_{i}} \Omega_{w}\right)=\sum_{t_{j k}} m_{q}\left(\Omega_{w \cdot t_{j k}}\right)+\sum_{t_{h l}} q_{h} \ldots q_{l-1} m_{q}\left(\Omega_{w \cdot t_{h l}}\right),
$$

where the first term is the one in the usual Monk formula, while the second term is summed over all transpositions $t_{h l}$ of integers $h \leq i<l$ such that $\ell\left(w \cdot t_{h l}\right)=$ $\ell(w)-2(l-h)+1$. 
Proof. We start by recalling some of the notation introduced earlier in the paper. Let $h, l$ be integers such that $1 \leq h \leq i \leq l \leq n-1$, and let $\bar{e}_{h l}$ be the multiindex $(0, \ldots, 0,1 \ldots, 1,0 \ldots, 0)$ defined in Lemma 3.9 .

Let $\mathcal{U}_{\bar{e}_{h l}} \subset X_{\bar{e}_{h l}}$ be the locally closed subset defined in Construction 2.2, and, as in 3.5, let $\mathcal{U}_{\bar{e}_{h l}}(u)$ be its restriction to $\pi^{-1}\left(\{u\} \times \mathcal{H} \mathcal{Q}_{\bar{d}-\bar{e}_{h l}}\right)$.

Let

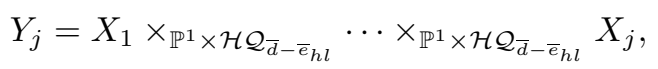

and let $p_{j}: Y_{j+1} \rightarrow Y_{j}$ and $\varrho_{j}: Y_{j+1} \rightarrow \mathbb{P}^{1} \times \mathcal{H} \mathcal{Q}_{\bar{d}_{-} \bar{e}_{h l}}$ be the projections. It is easily seen from the construction of $X_{\bar{e}_{h l}}$ that $p_{j}$ is the identity map for $j \in[0, n-2] \backslash[h-1, l-1]$, while for $h-1 \leq j \leq l-1$, the projection $p_{j}$ realizes $Y_{j+1}$ as the $\mathbb{P}^{j}$-bundle $\check{\mathbb{P}}\left(\varrho_{j-1}^{*} \mathcal{S}_{j+1}^{\bar{d}-\bar{e}_{h l}}\right)$ over $Y_{j}$. Recall that we have denoted in 2.4 by $U_{j}$ the locally closed subscheme of $Y_{j+1}$ determined by

$$
\operatorname{rank}\left(K_{j} \rightarrow V^{*}\right)=j, \quad j=1, \ldots, i+1,
$$

and

$$
\operatorname{rank}\left(K_{j} \rightarrow Q_{j+1}\right)=0, \quad j=1, \ldots, i .
$$

Let $U_{j}(u):=U_{j} \cap \varrho_{j}^{-1}\left(\{u\} \times \mathcal{H} \mathcal{Q}_{\bar{d}-\bar{e}_{h l}}\right)$. (In particular, $\mathcal{U}_{\bar{e}_{h l}}(u)=U_{n-2}(u)$, and $\varrho_{n-2}=\pi$.) One sees easily that the restriction

$$
p_{j}: U_{j}(u) \longrightarrow U_{j-1}(u)
$$

is a $\mathbb{P}^{1}$-bundle projection, for $h-1 \leq j \leq l-1$. More precisely,

$$
U_{j}(u) \cong \check{\mathbb{P}}\left(\varrho_{j-1}^{*} \mathcal{S}_{j+1}^{\bar{d}-\bar{e}_{h l}} / K_{j}\right) .
$$

Therefore the restriction

$$
\pi: \mathcal{U}_{\bar{e}_{h l}}(u) \longrightarrow\{u\} \times \mathcal{H} \mathcal{Q}_{\bar{d}-\bar{e}_{h l}}
$$

factors as a succession of $\mathbb{P}^{1}$-bundle projections

$$
\pi=p_{h-1} \circ \cdots \circ p_{l-1},
$$

and its image, $U_{0}(u)$, satisfies

$$
\{u\} \times H_{\bar{d}-\bar{e}_{h l}} \subset U_{0}(u) \subset\{u\} \times \mathcal{H} \mathcal{Q}_{\bar{d}_{-} \bar{e}_{h l}} .
$$

Let $w^{\prime} \in S_{n}$ be a permutation such that $\ell(w)+\ell\left(w^{\prime}\right)+1=n(n-1) / 2+\sum_{i=1}^{n-1} d_{i}$, let $u, t \in \mathbb{P}^{1}$ be distinct points, and let

$$
Z=\bar{\Omega}_{s_{i}}(u) \cap \bar{\Omega}_{w}(u) \cap \bar{\Omega}_{w^{\prime}}(t) .
$$

By Lemma $5.1(i)$ and Corollary 5.2, $Z$ is either empty or has pure dimension zero, and $\left\langle\Omega_{s_{i}}, \Omega_{w}, \Omega_{w^{\prime}}\right\rangle_{\bar{d}}=\operatorname{length}(Z)$. To evaluate this, we only have to look at

$$
Z \cap h_{\bar{e}_{h l}}\left(\mathcal{U}_{\bar{e}_{h l}}(u)\right),
$$

for all $1 \leq h \leq i \leq l \leq n-1$, by Lemma 5.1 (ii). Finally, by (iii) in Lemma 5.1, we may assume that $w$ satisfies

$$
w(h)>\max \{w(h+1), \ldots, w(l), w(l+1)\},
$$

and

$$
Z \cap h_{\bar{e}_{h l}}\left(\mathcal{U}_{\bar{e}_{h l}}(u)\right)=h_{\bar{e}_{h l}}\left(\tilde{\Omega}_{w}^{\bar{e}_{h l}}(u) \cap \pi^{-1}\left(\{u\} \times \bar{\Omega}_{w^{\prime}}(t)\right)\right) .
$$


Hence we need to decide when

$$
\tilde{\Omega}_{w}^{\bar{e}_{h l}}(u) \cap \pi^{-1}\left(\{u\} \times \bar{\Omega}_{w^{\prime}}(t)\right)
$$

is nonempty. Recall that by Construction 3.5 and Lemma 3.6, $\tilde{\Omega}_{w}^{\bar{e}_{h l}}(u)$ is defined inside $\mathcal{U}_{\bar{e}_{h l}}(u)$ by the conditions

$$
\begin{cases}\operatorname{rank}\left(V_{p} \otimes \mathcal{O} \rightarrow \pi^{*}\left(\mathcal{S}_{j}^{\bar{d}-\bar{e}_{h l}}\right)^{*}\right) \leq r_{\tilde{w}^{\bar{e}_{h l}}}(j, p), & j \in[1, n] \backslash[h, l], p \in[1, n], \\ \operatorname{rank}\left(V_{p} \otimes \mathcal{O} \rightarrow K_{j}^{*}\right) \leq r_{\tilde{w}^{\bar{e}_{h l}}}(j-1, p), & j \in[h, l], p \in[1, n],\end{cases}
$$

where $\tilde{w}^{\bar{e}_{h l}}$ is the permutation

$$
\left(\begin{array}{cccccccccc}
1 & \ldots & h-1 & h & \ldots & l & l+1 & l+2 & \ldots & n \\
w(1) & \ldots & w(h-1) & w(h+1) & \ldots & w(l+1) & w(h) & w(l+2) & \ldots & w(n)
\end{array}\right)
$$

from Lemma 3.9 (iii).

We consider first the projection

$$
p_{l-1}: U_{l-1}(u) \longrightarrow U_{l-2}(u) .
$$

If $w(l)<w(l+1)$, which means $\tilde{w}^{\bar{e}_{h l}}(l-1)<\tilde{w}^{\bar{e}_{h l}}(l)$, then the pairs $(l-1, p), 1 \leq$ $p \leq n$ are not in $\mathcal{E} s s\left(\tilde{w}^{\bar{e}_{h l}}\right)$. It follows that $p_{l-1}\left(\tilde{\Omega}_{w}^{\bar{e}} h l(u)\right)$ is the degeneracy locus in $U_{l-2}(u)$ associated to the sequence of (pull-backs of the) bundles

$$
\mathcal{S}_{1}^{\bar{d}-\bar{e}_{h l}}, \ldots, \mathcal{S}_{h-1}^{\bar{d}-\bar{e}_{h l}}, K_{h}, \ldots, K_{l-1}, \mathcal{S}_{l}^{\bar{d}-\bar{e}_{h l}}, \ldots, \mathcal{S}_{n-1}^{\bar{d}-\bar{e}_{h l}},
$$

(i.e., $K_{l}$, which doesn't live on $U_{l-2}(u)$, has been omitted), and the same permutation $\tilde{w}^{\bar{e}_{h l}}$. Its codimension is then equal to $\ell\left(\tilde{w}^{\bar{e}_{h l}}\right)$, and

$$
p_{l-1}\left(\tilde{\Omega}_{w}^{\bar{e}_{h l}}(u)\right) \cap\left(p_{h-1} \circ \cdots \circ p_{l-2}\right)^{-1}\left(\{u\} \times \bar{\Omega}_{w^{\prime}}(t)\right)
$$

is empty for dimension reasons. Therefore the same holds for (5.5.2).

However, if $w(l)>w(l+1)$, then it follows from the exact sequence

$$
0 \rightarrow \mathcal{O}(-1) \rightarrow\left(p_{l-1}^{*} \mathcal{S}_{l}^{\bar{d}-\bar{e}_{h l}} / K_{l-1}\right)^{*} \rightarrow\left(K_{l} / K_{l-1}\right)^{*} \rightarrow 0
$$

on the $\mathbb{P}^{1}$-bundle

$$
p_{l-1}: U_{l-1}(u) \longrightarrow U_{l-2}(u)
$$

that $p_{l-1}\left(\tilde{\Omega}_{w}^{\bar{e}_{h l}}(u)\right)$ is given inside $U_{l-2}(u)$ by the rank conditions (5.5.3), except that

$$
\operatorname{rank}\left(V_{p} \otimes \mathcal{O} \rightarrow K_{l}^{*}\right) \leq r_{\tilde{w}^{e_{h l}}}(l-1, p), \quad 1 \leq p \leq n,
$$

are changed into

$$
\operatorname{rank}\left(V_{p} \otimes \mathcal{O} \rightarrow\left(\mathcal{S}_{l}^{\bar{d}-\bar{e}_{h l}}\right)^{*}\right) \leq\left\{\begin{array}{l}
r_{\tilde{w}^{\bar{e}_{h l}}}(l-1, p), \text { if } 1 \leq p<\tilde{w}^{\bar{e}_{h l}}(l) \\
r_{\tilde{w}^{\bar{e}} h l}(l-1, p)+1, \text { if } \tilde{w}^{\bar{e}_{h l}}(l) \leq p \leq n .
\end{array}\right.
$$

We can immediately verify that this new collection of rank numbers is permissible, the corresponding permutation being $\tilde{w}^{\bar{e}_{h l}} \cdot s_{l-1}$. In this case the restriction of $p_{l-1}$ to $\tilde{\Omega}_{w}^{\bar{e}_{w l}}(u)$ is birational onto its image, and the sum of the codimensions of $p_{l-1}\left(\tilde{\Omega}_{w}^{\bar{e}_{h l}}(u)\right)$ and $\left(p_{h-1} \circ \cdots \circ p_{l-2}\right)^{-1}\left(\{u\} \times \bar{\Omega}_{w^{\prime}}(t)\right)$ does equal the dimension of $U_{l-2}(u)$. The same argument can now be applied successively to the projections $p_{l-2}, \ldots, p_{h-1}$ to conclude that either (5.5.2) is empty or

$$
w(l+1)<\min \{w(h+1), \ldots, w(l)\},
$$


the restriction of $\pi$ to $\tilde{\Omega}_{w}^{\bar{e}_{h l}}(u)$ is birational onto its image, and

$$
\pi\left(\tilde{\Omega}_{w}^{\bar{e}_{h l}}(u)\right)=\bar{\Omega}_{w \cdot t_{h l+1}}(u) \cap U_{h-1}(u),
$$

where $t_{h l+1}$ is the transposition interchanging $h$ and $l+1$ (we have used here that $\left.\tilde{w}^{\bar{e}_{h l}} \cdot s_{l-1} \cdots s_{h}=w \cdot t_{h l+1}\right)$. By Lemma 4.5 ,

$$
\bar{\Omega}_{w \cdot t_{h l+1}}(u) \cap\{u\} \times \bar{\Omega}_{w^{\prime}}(t)
$$

is empty (hence (5.5.2) is empty as well) unless $\bar{d}=\bar{e}_{h l}$, and $\Omega_{w^{\prime}}$ is the complementary Schubert cycle to $\Omega_{w \cdot t_{h l+1}}$. In this case

$$
\mathcal{U}_{\bar{e}_{h l}}(u)=\pi^{-1}(\{u\} \times F),
$$

and, by Theorem $2.3(i i)(b), h_{\bar{e}_{h l}}$ is an isomorphism onto its image. Therefore

$$
\operatorname{length}(Z)=\operatorname{length}\left(\tilde{\Omega}_{w}^{\bar{e}_{h l}}(u)\right) \cap \pi_{\bar{d}-\bar{e}_{h l}}^{-1}\left(\{u\} \times \bar{\Omega}_{w^{\prime}}(t)\right),
$$

and the latter can be computed as

$$
\operatorname{deg}\left(\tilde{\Omega}_{w}^{\bar{e}_{h l}}(u) \cdot \pi^{*}\{u\} \times \bar{\Omega}_{w^{\prime}}(t)\right)
$$

in the Chow ring of $\pi^{-1}(\{u\} \times F)$. By the projection formula,

$$
\pi_{*}\left(\tilde{\Omega}_{w}^{\bar{e}_{h l}}(u) \cdot \pi^{*}\{u\} \times \bar{\Omega}_{w^{\prime}}(t)\right)=\pi_{*}\left(\tilde{\Omega}_{w}^{\bar{e}_{h l}}(u)\right) \cdot\left(\{u\} \times \bar{\Omega}_{w^{\prime}}(t)\right),
$$

and from $(5.5 .6)$

$$
\pi_{*}\left(\tilde{\Omega}_{w}^{\bar{e}_{h l}}(u)\right) \cdot\{u\} \times \bar{\Omega}_{w^{\prime}}(t)=\bar{\Omega}_{w \cdot t_{h l+1}}(u) \cdot\{u\} \times \bar{\Omega}_{w^{\prime}}(t)=\Omega_{w \cdot t_{h l+1}} \cdot \Omega_{w^{\prime}}=1,
$$

the products in the previous line being all computed in the Chow ring of $F$. Summarizing, we have proven that

$$
\left\langle\Omega_{s_{i}}, \Omega_{w}, \Omega_{w^{\prime}}\right\rangle_{\bar{d}}=0,
$$

unless $\bar{d}=\bar{e}_{h l}, w$ satisfies (5.5.1) and (5.5.5), and $\Omega_{w^{\prime}}$ is the complementary Schubert cycle to $\Omega_{w \cdot t_{h l+1}}$, in which case

$$
\left\langle\Omega_{s_{i}}, \Omega_{w}, \Omega_{w^{\prime}}\right\rangle_{\bar{e}_{h l}}=1 .
$$

Replacing $l+1$ by $l$, this gives exactly the formula we were seeking.

Finally, we can give now a presentation by generators and relations for the quantum cohomology ring.

For all $1 \leq k \leq m \leq n$ we denote by $R_{k}(m)$ the $k^{\text {th }}$ elementary symmetric function in the variables $x_{1}, \ldots, x_{m}$. Also, denote by $A_{k}(m)$ the class corresponding to $R_{k}(m)$ in the cohomology of $F$. Notice that $A_{k}(n)=0$ for all $1 \leq k \leq n$, since

$$
H^{*}(F ; \mathbb{Z}) \cong \mathbb{Z}\left[x_{1}, x_{2}, \ldots, x_{n}\right] /\left(R_{1}(n), R_{2}(n), \ldots, R_{n}(n)\right) .
$$

In the quantum cohomology ring however, $m_{q}\left(R_{k}(n)\right)$ does not vanish anymore. In fact, consider for $k \leq m \leq n$ the following polynomials in $\mathbb{Z}\left[x_{1}, \ldots, x_{n}, q_{1}, \ldots, q_{n-1}\right]$ :

$$
\begin{aligned}
R_{k}^{\prime}(m) & :=\sum_{i=1}^{m-1} q_{i}\left(\frac{\partial^{2}}{\partial x_{i} \partial x_{i+1}} R_{k}(m)\right)+\sum_{i<j} q_{i} q_{j}\left(\frac{\partial^{4}}{\partial x_{i} \partial x_{i+1} \partial x_{j} \partial x_{j+1}} R_{k}(m)\right) \\
& +\cdots+\sum_{i_{1}<\cdots<i_{\left[\frac{k}{2}\right]}} q_{i_{1}} \ldots q_{i_{\left[\frac{k}{2}\right]}}\left(\frac{\partial^{2\left[\frac{k}{2}\right]}}{\partial x_{i_{1}} \partial x_{i_{1}+1} \ldots \partial x_{i_{\left[\frac{k}{2}\right]}} \partial x_{i_{\left[\frac{k}{2}\right]+1}}} R_{k}(m)\right)
\end{aligned}
$$

( $\left[\frac{k}{2}\right]$ denotes as usual the greatest integer $\left.\leq \frac{k}{2}\right)$. Then we have 
Theorem 5.6. (i) For all $1 \leq k \leq m \leq n$,

$$
m_{q}\left(R_{k}(m)\right)=m_{q}\left(A_{k}(m)\right)-m_{q}\left(R_{k}^{\prime}(m)\right) .
$$

In particular $m_{q}\left(R_{k}(n)\right)+m_{q}\left(R_{k}^{\prime}(n)\right)=0$ for $k=1, \ldots, n$, i.e. the polynomials

$$
R_{k}^{q}(n):=R_{k}(n)+R_{k}^{\prime}(n)
$$

are in the ideal $I_{q}$.

(ii) $I_{q}$ is generated by $R_{1}^{q}(n), \ldots, R_{n}^{q}(n)$.

Proof. (i) We will proceed by induction on $m$.

First notice that for $k=1$ we have $R_{1}^{\prime}(m)=0$ for all $1 \leq m \leq n$ and therefore the formula $(*)$ follows from the fact that $m_{q}$ is the identity map on $\operatorname{Sym}^{1}\left(H^{*}(F ; \mathbb{Z})\left[q_{1}, \ldots, q_{n-1}\right]\right)$ (Lemma 4.5). This also takes care of the case $m=1$.

We assume now that $\left(^{*}\right)$ is true for all $k$ and $m^{\prime}$ with $1 \leq k \leq m^{\prime}<m$. Since $m_{q}$ is linear,

$$
m_{q}\left(R_{k}(m)\right)=m_{q}\left(R_{k}(m-1)\right)+m_{q}\left(x_{m} R_{k-1}(m-1)\right) .
$$

By the induction hypothesis,

$$
\begin{aligned}
m_{q}\left(R_{k}(m)\right) & =m_{q}\left(A_{k}(m-1)\right)-m_{q}\left(R_{k}^{\prime}(m-1)\right) \\
& +m_{q}\left(x_{m} A_{k-1}(m-1)\right)-m_{q}\left(x_{m} R_{k-1}^{\prime}(m-1)\right) .
\end{aligned}
$$

Recall the cycle $\alpha_{k, m}, m \leq n, k \leq m-1$. Its Schubert polynomial is the $k-1^{\text {st }}$ elementary symmetric function in variables $x_{1}, \ldots, x_{m-1}$,

$$
\mathfrak{S}_{\alpha_{k, m}}(x)=R_{k-1}(m-1),
$$

as can be easily seen. Hence $A_{k-1}(m-1)=\Omega_{\alpha_{k, m}}$ in $H^{*}(F ; \mathbb{Z})$, by Theorem 0.3 .

Recall also that $x_{m}=\Omega_{s_{m}}-\Omega_{s_{m-1}}$, for all $1 \leq m \leq n$, with the convention $\Omega_{s_{m}}=0$, for $m=0$ or $n$. The special case of the quantum Monk formula (Proposition 5.3) gives now

$$
\begin{aligned}
m_{q}\left(x_{m} A_{k-1}(m-1)\right) & =m_{q}\left(\Omega_{s_{m}} \Omega_{\alpha_{k, m}}\right)-m_{q}\left(\Omega_{s_{m-1}} \Omega_{\alpha_{k, m}}\right) \\
& =m_{q}\left(B_{k, m}\right)-q_{m-1} m_{q}\left(\Omega_{\alpha_{k, m} \cdot s_{m-1}}\right),
\end{aligned}
$$

where by $B_{k, m}$ we have denoted the class of $x_{m} \cdot A_{k-1}(m-1)$ in $H^{*}(F ; \mathbb{Z})$.

Obviously, $B_{k, m}=A_{k}(m)-A_{k}(m-1)$. Moreover, since $\alpha_{k, m} \cdot s_{m-1}=\alpha_{k-1, m-1}$, we can use again Theorem 0.3 to conclude that $\Omega_{\alpha_{k, m} \cdot s_{m-1}}=A_{k-2}(m-2)$. With these observations (5.6.2) becomes

$$
m_{q}\left(x_{m} A_{k-1}(m-1)\right)=m_{q}\left(A_{k}(m)-A_{k}(m-1)\right)-q_{m-1} m_{q}\left(A_{k-2}(m-2)\right) .
$$

By the inductive assumption,

$$
m_{q}\left(A_{k-2}(m-2)\right)=m_{q}\left(R_{k-2}(m-2)+R_{k-2}^{\prime}(m-2)\right) .
$$

Combining (5.6.1), (5.6.3), (5.6.4), and the easy polynomial identity

$$
R_{k}^{\prime}(m)=R_{k}^{\prime}(m-1)+x_{m} R_{k-1}^{\prime}(m-1)+q_{m-1} R_{k-2}(m-2)+q_{m-1} R_{k-2}^{\prime}(m-2),
$$

we get the formula $(*)$.

(ii) is a consequence of $(i)$ and [ST, Theorem 2.2]. 
In [GK], Givental and Kim conjectured that the generators for the ideal $I_{q}$ are the coefficients $C_{k}(n)$ of $\lambda^{n-k}, k=1, \ldots, n$ in the expansion of the determinant:

$$
\left|\begin{array}{ccccccc}
x_{1}+\lambda & q_{1} & 0 & 0 & \ldots & 0 & 0 \\
-1 & x_{2}+\lambda & q_{2} & 0 & \ldots & 0 & 0 \\
0 & -1 & x_{3}+\lambda & q_{3} & \ldots & 0 & 0 \\
\vdots & \vdots & \vdots & \vdots & \vdots & \vdots & \vdots \\
0 & 0 & 0 & 0 & \ldots & x_{n-1}+\lambda & q_{n-1} \\
0 & 0 & 0 & 0 & \ldots & -1 & x_{n}+\lambda
\end{array}\right| .
$$

The following lemma shows that this is the same as the presentation of Theorem 5.6 .

Lemma 5.7. $C_{k}(n)=R_{k}^{q}(n)$, for all $k=1, \ldots, n$.

Proof. The polynomials $R_{k}^{q}(n)$ satisfy the recursion

$$
R_{k}^{q}(n)=R_{k}^{q}(n-1)+x_{n} R_{k-1}^{q}(n-1)+q_{n-1} R_{k-2}^{q}(n-2)
$$

(combine (5.6.5) above with the recursion $R_{k}(n)=R_{k}(n-1)+x_{n} R_{k-1}(n-1)$ satisfied by the elementary symmetric polynomials). By expanding the GiventalKim determinant along the last column, one sees that the polynomials $C_{k}(n)$ satisfy the same recursion, and the lemma follows.

\section{ApPENDIX: EXISTENCE AND LOCAL PROPERTIES OF HYPERQUOT SCHEMES}

The proofs we give here are taken from [C-F2]. Although rather lengthy, they are straightforward following the same line as for the corresponding results in the case of the Quot functor. The ideas are due to Grothendieck ([Gr]). We follow the presentation by Kollár ([Kol]), adapted to our case.

As usual, the following two general results will be used in an essential way. The proofs in [Mu, Lectures 14 and 8] require only minor modifications.

Theorem A. Let $X$ be a projective variety, $\mathcal{E}$ be a vector bundle on $X$ and $\mathcal{G}$ be a subsheaf of $\mathcal{E}$ with fixed Hilbert polynomial $P$. Then there exists an integer $N$, depending only on $P$, such that for every $m \geq N$ the following hold:

(A1) $H^{i}(X, \mathcal{G}(m))=0$ for $i \geq 1$.

(A2) $\mathcal{G}(m)$ is generated by global sections.

Theorem B. Let $X$ be a projective scheme and let $S$ be any scheme. Let $\mathcal{G}$ be a coherent sheaf on $X \times S$. For every numerical polynomial $P$ there exists a locally closed subscheme $i_{P}: S_{P} \hookrightarrow S$ with the following property:

Given any morphism $g: T \longrightarrow S$, the pull-back $(i d, g)^{*} \mathcal{G}$ on $X \times T$ is flat over $T$ with Hilbert polynomial $P$ if and only if $g$ can be factored as

$$
g: T \longrightarrow S_{P} \stackrel{i_{P}}{\longrightarrow} S .
$$

Proof of Theorem 1.1. Given $\mathcal{E}, n$ and $\bar{P}=\left(P_{1}, \ldots, P_{n-1}\right)$, it follows from Theorem A that there is an $N$ such that for every sequence of flat quotients

$$
\pi^{*} \mathcal{E} \rightarrow Q_{n-1} \rightarrow \cdots \rightarrow Q_{1}
$$

with $\chi\left(X_{s}, Q_{j_{s}}(m)\right)=P_{j}(m)$, if we let

$$
\mathcal{E}_{j}=\operatorname{ker}\left\{\pi^{*} \mathcal{E} \rightarrow Q_{n-j}\right\},
$$


then $H^{i}\left(X_{s}, \mathcal{E}_{j_{s}}(a)\right)=0$ for all $i \geq 1, a \geq N, s \in S$ and also $\mathcal{E}_{j_{s}}(a)$ is generated by global sections. (Note that each $\mathcal{E}_{j}$ is flat.)

We may choose $N$ large enough so that $\mathcal{E}(N)$ is generated by global sections and we have $H^{i}(X, \mathcal{E}(N))=0$ for $i \geq 1$ as well, hence $H^{i}\left(X_{s}, \mathcal{E}_{s}(N)\right)=0$ for all $s \in S$.

Since all $Q_{j}$ are flat over $S$, the sequences

$$
0 \rightarrow \mathcal{E}_{j_{s}}(N) \rightarrow \mathcal{E}_{s}(N) \rightarrow Q_{(n-j)_{s}}(N) \rightarrow 0
$$

are all exact for every $s \in S$, so we see that $H^{i}\left(X_{s}, Q_{j_{s}}(N)\right)=0$ for $i \geq 1$.

Let $f: X \times S \longrightarrow S$ be the projection. Let $Y$ be the partial flag variety parametrizing successive quotients

$$
H^{0}(X, \mathcal{E}(N)) \rightarrow G_{n-1} \cdots \rightarrow G_{1},
$$

with $\operatorname{rank}\left(G_{j}\right)=P_{j}(N)$. The discussion above implies that the sheaf $f_{*} Q_{n-j}(N)$ is locally free of $\operatorname{rank} P_{n-j}(N)$, for each $j=1, \ldots, n-1$. Moreover, there are surjective maps

$$
H^{0}(X, \mathcal{E}(N)) \otimes \mathcal{O}_{S}=f_{*} \pi_{X}^{*} \mathcal{E}(N) \rightarrow f_{*} Q_{n-1}(N) \cdots \rightarrow f_{*} Q_{1}(N) .
$$

Thus each element of $\mathcal{F}_{n, \bar{P}}(X, \mathcal{E})(S)$ determines a morphism $\Phi: S \longrightarrow Y$ and so we get a morphism of functors $\mathcal{F}_{n, \bar{P}}(X, \mathcal{E}) \longrightarrow h_{Y}$, where $h_{Y}$ is the functor of points associated to $Y$. Let

$$
\mathcal{U}_{1} \hookrightarrow \cdots \hookrightarrow \mathcal{U}_{n-1} \hookrightarrow H^{0}(X, \mathcal{E}(N)) \otimes \mathcal{O}_{Y}
$$

be the universal sequence of subbundles on $Y$. Consider the two projections

$$
Y \stackrel{\pi_{1}}{\longleftarrow} Y \times X \stackrel{\pi_{2}}{\longrightarrow} X
$$

and form the compositions

$$
g_{j}: \pi_{1}^{*} \mathcal{U}_{j} \longrightarrow H^{0}(X, \mathcal{E}(N)) \otimes \mathcal{O}_{X \times Y} \longrightarrow \pi_{2}^{*} \mathcal{E}(N) .
$$

Let $\mathcal{K}_{j}=\operatorname{coker}\left(g_{j}\right), j=1, \ldots, n-1$. By Theorem B applied to $\mathcal{K}_{j}(-N)$, there is a largest locally closed subscheme $Z_{P_{n-j}} \stackrel{i_{j}}{\hookrightarrow} Y$ with the property that $i_{j}^{*} \mathcal{K}_{j}(-N)$, as sheaf on $Z_{P_{n-j}} \times X$, is flat over $Z_{P_{n-j}}$ with Hilbert polynomial $P_{n-j}(m)$. Let $Z_{\bar{P}}$ be the scheme-theoretic intersection of $Z_{P_{1}}, \ldots, Z_{P_{n-1}}$. We claim that $\mathcal{F}_{n, \bar{P}}(X, \mathcal{E})$ is represented by $Z_{\bar{P}}$. Indeed, given a sequence of flagged quotients on $X \times S$

$$
\pi^{*} \mathcal{E} \rightarrow Q_{n-1} \rightarrow \cdots \rightarrow Q_{1},
$$

flat over $S$ with Hilbert polynomials $P_{n-1}(m), \ldots, P_{1}(m)$ respectively, we have seen that there is a morphism $\Phi: S \longrightarrow Y$ such that

$$
\Phi^{*}\left[\mathcal{U}_{j} \rightarrow H^{0}(X, \mathcal{E}(N)) \otimes \mathcal{O}_{Y}\right] \cong\left[f_{*} \mathcal{E}_{j}(N) \rightarrow H^{0}(X, \mathcal{E}(N)) \otimes \mathcal{O}_{S}\right] .
$$

From the diagram

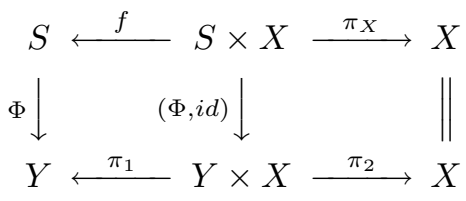


we get

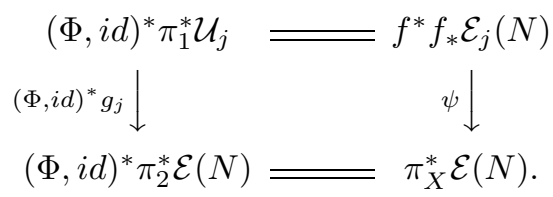

But $\operatorname{Im} \psi=\mathcal{E}_{j}(N)$, since $\mathcal{E}_{j_{s}}(N)$ are generated by sections for every $s \in S$. This implies that $(\Phi, i d)^{*} \mathcal{K}_{j} \cong Q_{n-j}(N)$. Hence $(\Phi, i d)^{*} \mathcal{K}_{j}(-N)$ are all flat over $S$ with Hilbert polynomials $P_{n-j}$. Therefore $\Phi$ factors through $Z_{\bar{P}}$ and we see that there is an injective morphism of functors $p: \mathcal{F}_{n, \bar{P}}(X, \mathcal{E}) \longrightarrow h_{Z_{\bar{P}}}$. By construction, every morphism $S \longrightarrow Z_{\bar{P}}$ gives an element of $\mathcal{F}_{n, \bar{P}}(X, \mathcal{E})(S)$. This provides an inverse for $p$ and proves the claim.

As for the projectivity, we only need to check that $Z_{\bar{P}}$ is proper. But this follows at once from the valuative criterion (using the case of the Quot scheme).

For the proof of Theorem 1.2 we shall use the standard techniques of deformation theory. For the convenience of the reader we reproduce part of the exposition in [Kol, Sec. I.2].

We treat first the local case. Let $\left(A, m_{A}\right)$ and $\left(B, m_{B}\right)$ be local Artin rings with residue field $k$ such that $B$ is an extension of $A$ by the ideal $J$ :

$$
0 \rightarrow J \rightarrow B \rightarrow A \rightarrow 0,
$$

with $m_{B} J=0$. Let $T$ be a $k$-algebra and let $T^{B}$ be a Noetherian ring flat over $B$ such that $T^{B} \otimes_{B} k \cong T$. Let $T^{A}=T^{B} \otimes_{B} A$. Denote by $M=M^{k}$ the free rank $r T$-module and let $F^{k}=F \stackrel{j}{\hookrightarrow} M$ be a submodule. Let $Q=M / F$ and let $p: M \rightarrow Q$ be the projection. Let

$$
R \stackrel{r}{\longrightarrow} G \stackrel{g}{\longrightarrow} F \longrightarrow 0
$$

be a presentation of $F$ by free $T$-modules. In what follows the extensions of the above $T$-modules and morphisms to $T^{A}$ and $T^{B}$ will receive corresponding upper indices.

Let $F^{A} \stackrel{j^{A}}{\longrightarrow} M^{A}$ be such that $Q^{A}=M^{A} / F^{A}$ is flat over $A$ and $F^{A} \otimes_{A} k=F$. Flatness implies that the presentation of $F$ lifts to a presentation of $F^{A}$ :

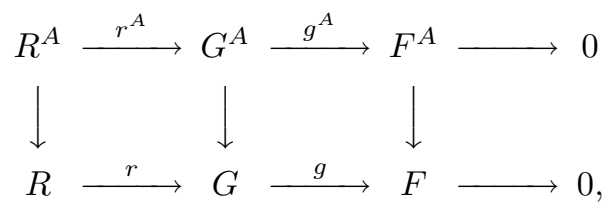

where the vertical arrows are specializations. Pick arbitrary liftings $g^{B}: G^{B} \rightarrow$ $M^{B}$ and $r^{B}: R^{B} \rightarrow G^{B}$ (recall that $R, G, M$ are free modules). This gives a commutative diagram

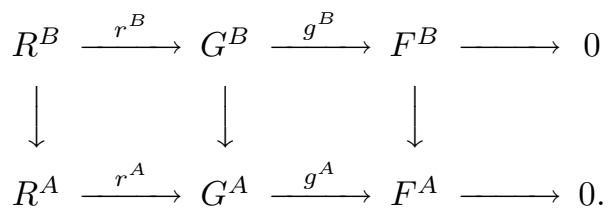


Here $F^{B} \subset M^{B}$ is the image of $G^{B}$. Note that the top row need not be a presentation and that the quotient $Q^{B}=M^{B} / F^{B}$ need not be flat. The following result (Lemma I.2.2 in [Kol]) gives the conditions under which the lifting $Q^{B}$ is flat.

Lemma C. $(i)$ To the diagram $\left(^{*}\right)$ one can associate a morphism $e\left(g^{B}\right): R \longrightarrow$ $Q \otimes J$, which is independent of the choice of $r^{B}$.

(ii) The module $E\left(g^{B}\right):=\operatorname{coker}\left[R \stackrel{e\left(g^{B}\right)+r}{\longrightarrow} Q \otimes J+G\right]$ can be written as an extension

$$
0 \rightarrow Q \otimes J \rightarrow E\left(g^{B}\right) \rightarrow F \rightarrow 0,
$$

hence we have an element $\left[E\left(g^{B}\right)\right] \in \operatorname{Ext}_{T}^{1}(F, Q \otimes J)$.

(iii) Any different lifting $\bar{g}^{B}: G^{B} \rightarrow M^{B}$ can be written as $\bar{g}^{B}=g^{B}+\phi^{B}$, where $\phi^{B} \in \operatorname{Hom}\left(G^{B}, M \otimes J\right)$. This gives a natural extension isomorphism

$$
E\left(\phi^{B}\right): E\left(g^{B}\right) \longrightarrow E\left(\bar{g}^{B}\right) .
$$

In particular, $\left[E\left(g^{B}\right)\right] \in \operatorname{Ext}_{T}^{1}(F, Q \otimes J)$ is independent of the choice of $g^{B}$ and depends only on the data over $A$. It is denoted by $\left[E^{B}\left(Q^{A}\right)\right]$ and called the obstruction.

(iv) The following are equivalent:

(a) $Q^{B}$ is flat over $B$.

(b) $e\left(g^{B}\right)=0$.

(c) $g^{B} \circ r^{B}=0$ for a suitable choice of $r^{B}$ (i.e. the top row in $\left(^{*}\right)$ is a complex).

(v) For a suitable choice of $g^{B}$, the quotient $Q^{B}$ is flat over $B$ if and only if $\left[E^{B}\left(Q^{A}\right)\right]=0$. In this case, there are affine linear isomorphisms

$$
\left\{\begin{array}{c}
\text { set of quotients } Q^{B} \\
\text { which are flat over } B
\end{array}\right\} \cong\{\text { splittings of }(\dagger)\} \cong \operatorname{Hom}_{T}(F, Q \otimes J) \text {. }
$$

(vi) If $R_{1}^{A} \stackrel{r_{1}^{A}}{\longrightarrow} G_{1}^{A} \stackrel{g_{1}^{A}}{\longrightarrow} F^{A} \longrightarrow 0$ is a different presentation of $F^{A}$, then there is a natural extension isomorphism $E\left(g^{B}\right) \cong E\left(g_{1}^{B}\right)$. Thus $\left[E^{B}\left(Q^{A}\right)\right]$ depends only on $Q^{A}$. Also, if $\left[E^{B}\left(Q^{A}\right)\right]=0$, then the principal homogeneous space structure on the set of quotients $Q^{B}$ which are flat over $B$ depends on $Q^{A}$ only.

Remark $D$. The following special case will be used later.

Take $A=k$ and $B=k+J$. Then

$$
T^{B}=T^{B} \otimes_{B} k+T^{B} \otimes_{B} J=T+T \otimes_{k} J .
$$

In this case there is always the trivial flat lifting of $F^{k}$ to $F_{\text {triv }}^{B}=F^{k}+F^{k} \otimes_{k} J$. This implies that we have a natural isomorphism

$$
\left\{\begin{array}{c}
\text { set of quotients } Q^{B} \\
\text { which are flat over } B
\end{array}\right\} \cong \operatorname{Hom}_{T}(F, Q \otimes J)
$$

with the trivial extension as the zero vector. We will be interested in lifting successive quotients

$$
M^{A} \stackrel{p_{n-1}}{\rightarrow} Q_{n-1}^{A} \stackrel{p_{n-2}}{\rightarrow} Q_{n-2}^{A} \rightarrow \ldots \stackrel{p_{1}}{\rightarrow} Q_{1}^{A} .
$$

Let $F_{i}^{A}:=\operatorname{ker}\left(M^{A} \rightarrow Q_{n-i}^{A}\right)$ and denote by $F_{i}^{A} \stackrel{j_{i}}{\longleftrightarrow} F_{i+1}^{A}$ the inclusions. Let

$$
K:=\operatorname{ker}\left[\bigoplus_{i=1}^{n-1} \operatorname{Hom}_{T}\left(F_{i}, Q_{n-i} \otimes J\right) \stackrel{f}{\longrightarrow} \bigoplus_{i=1}^{n-2} \operatorname{Hom}_{T}\left(F_{i}, Q_{n-i-1} \otimes J\right)\right],
$$


where $f$ is defined by $f\left(\left\{\phi_{i}\right\}_{i=1}^{n-1}\right)=\left\{p_{n-i-1} \circ \phi_{i}-\phi_{i+1} \circ j_{i}\right\}_{i=1}^{n-2}$. Then Lemma C $(v)$ implies that

$$
\left\{\begin{array}{c}
\text { set of successive quotients } \\
\left\{Q_{i}^{B}\right\} \text { which are flat over } B
\end{array}\right\} \cong K
$$

We will pass now to the global situation.

Let $X$ be a smooth projective variety, let $\mathcal{E}$ be a rank $r$ vector bundle on $X$, and let $\bar{P}=\left(P_{1}(m), \ldots, P_{n-1}(m)\right)$ be numerical polynomials. Assume that we are given a sequence of quotient sheaves

$$
\mathcal{E} \stackrel{p_{n-1}}{\rightarrow} \mathcal{Q}_{n-1} \stackrel{p_{n-2}}{\rightarrow} \mathcal{Q}_{n-2} \rightarrow \ldots \stackrel{p_{1}}{\rightarrow} \mathcal{Q}_{1}
$$

with $\chi\left(X, \mathcal{Q}_{j}(m)\right)=P_{j}(m)$ and let $x \in \mathcal{H} Q_{n, \bar{P}}(X, \mathcal{E})$ be the corresponding point. Let

$$
\mathcal{E}_{i}=\operatorname{ker}\left(\mathcal{E} \rightarrow \mathcal{Q}_{n-i}\right)
$$

and denote by $\mathcal{E}_{i} \stackrel{j_{i}}{\longrightarrow} \mathcal{E}_{i+1}$ the corresponding sheaf inclusions.

Consider the map of sheaves

$$
\bigoplus_{i=1}^{n-1} \mathcal{H o m}_{\mathcal{O}_{X}}\left(\mathcal{E}_{i}, \mathcal{Q}_{n-i}\right) \stackrel{f}{\longrightarrow} \bigoplus_{i=1}^{n-2} \mathcal{H o m}_{\mathcal{O}_{X}}\left(\mathcal{E}_{i}, \mathcal{Q}_{n-i-1}\right),
$$

given ( for any open set $U \subset X$ ) by

$$
f\left(\left\{\phi_{i}\right\}_{i=1}^{n-1}\right)=\left\{p_{n-i-1} \circ \phi_{i}-\phi_{i+1} \circ j_{i}\right\}_{i=1}^{n-2}
$$

and let $\mathcal{K}:=\operatorname{ker} f$.

The following proposition is the main step for the proof of Theorem 1.2 (compare with [Kol, Prop. I.2.5 and Thm I.2.8] ).

Proposition E ([Lau1, Prop. 2.5]). (i) The Zariski tangent space to $\mathcal{H} \mathcal{Q}_{n, \bar{P}}(X, \mathcal{E})$ at $x$ is isomorphic to $H^{0}(X, \mathcal{K})$.

(ii) Assume in addition that $\operatorname{Ext}^{1}\left(\mathcal{E}_{i}, \mathcal{Q}_{n-i}\right)=0$, for $i=1, \ldots, n-1$. If $H^{1}(X, \mathcal{K})=0$, then $\mathcal{H} \mathcal{Q}_{n, \bar{P}}(X, \mathcal{E})$ is smooth at $x$.

Proof. Let $U_{\alpha}=\operatorname{Spec}\left(T_{\alpha}\right)$ be an affine covering of $X$ such that $\left.\mathcal{E}\right|_{U_{\alpha}}$ is trivial. Denote by $F_{i \alpha}^{k}, M_{\alpha}^{k}, Q_{i \alpha}^{k}, K_{\alpha}^{k}$ the $T_{\alpha}$-modules obtained by restricting $\mathcal{E}_{i}, \mathcal{E}, \mathcal{Q}_{i}$ and $\mathcal{K}$ respectively to $U_{\alpha}$.

To prove $(i)$ we let $A=k, B=k[\epsilon] / \epsilon^{2}$. Let $\mathcal{E}^{B}$ be the pull-back of $\mathcal{E}$ to $X \times \operatorname{Spec}(B)$. The tangent space to $\mathcal{H} \mathcal{Q}$ at $x$ is given by the set of all liftings

$$
\mathcal{E}^{B} \stackrel{p_{n-1}^{B}}{\rightarrow} \mathcal{Q}_{n-1}^{B} \stackrel{p_{n-2}^{B}}{\rightarrow} \mathcal{Q}_{n-2}^{B} \rightarrow \ldots \stackrel{p_{1}^{B}}{\rightarrow} \mathcal{Q}_{1}^{B}
$$

which are flat over $\operatorname{Spec}(B)$ and specialize to

$$
\mathcal{E} \stackrel{p_{n-1}}{\rightarrow} \mathcal{Q}_{n-1} \stackrel{p_{n-2}}{\rightarrow} \mathcal{Q}_{n-2} \rightarrow \ldots \stackrel{p_{1}}{\rightarrow} \mathcal{Q}_{1}
$$

over $X \times \operatorname{Spec}(k)$.

As we remarked earlier, we can always take over $U_{\alpha}$ the trivial liftings $Q_{i \alpha}^{B}=$ $Q_{i \alpha}^{k} \otimes_{k} B$ and they glue together to give trivial liftings $\mathcal{Q}_{i}^{B}$, flat over $B$ and such that the maps $\mathcal{Q}_{i+1}^{B} \rightarrow \mathcal{Q}_{i}^{B}$ are all surjective. By Remark D, any other flat lifting of the successive quotients is obtained from elements $\phi_{\alpha} \in H^{0}\left(U_{\alpha}, K_{\alpha}\right)$ which glue together. This gives $(i)$. 
(ii) Take $B \rightarrow A$ to be a quotient of local Artin rings with residue fields equal to $k$. It is well known that the local $\operatorname{ring} \mathcal{O}_{x, \mathcal{H}}$ of $\mathcal{H Q}$ at $x$ is regular if and only if, for every such quotient, the induced map $\operatorname{Hom}_{k}\left(\mathcal{O}_{x, \mathcal{H}}, B\right) \rightarrow \operatorname{Hom}_{k}\left(\mathcal{O}_{x, \mathcal{H}}, A\right)$ is surjective. This says that we need to check the following assertion:

For every sequence of flagged quotients

$$
\mathcal{E}^{A} \stackrel{p_{n-1}^{A}}{\rightarrow} \mathcal{Q}_{n-1}^{A} \stackrel{p_{n-2}^{A}}{\rightarrow} \mathcal{Q}_{n-2}^{A} \rightarrow \ldots \stackrel{p_{1}^{A}}{\rightarrow} \mathcal{Q}_{1}^{A}
$$

on $X \times \operatorname{Spec}(A)$, which are flat over $\operatorname{Spec}(A)$ and specialize to

$$
\mathcal{E} \stackrel{p_{n-1}}{\rightarrow} \mathcal{Q}_{n-1} \stackrel{p_{n-2}}{\rightarrow} \mathcal{Q}_{n-2} \rightarrow \ldots \stackrel{p_{1}}{\rightarrow} \mathcal{Q}_{1}
$$

over $X \times \operatorname{Spec}(k)$, there exists a lifting

$$
\mathcal{E}^{B} \stackrel{p_{n-1}^{B}}{\rightarrow} \mathcal{Q}_{n-1}^{B} \stackrel{p_{n-2}^{B}}{\rightarrow} \mathcal{Q}_{n-2}^{B} \rightarrow \ldots \stackrel{p_{1}^{B}}{\rightarrow} \mathcal{Q}_{1}^{B}
$$

such that each $\mathcal{Q}_{i}^{B}$ is flat over $\operatorname{Spec}(B)$.

Pick presentations

$$
R_{i \alpha}^{A} \rightarrow G_{i \alpha}^{A} \rightarrow F_{i \alpha}^{A} \rightarrow 0
$$

of the restrictions of the sheaves $\mathcal{E}_{i}^{A}$ to $U_{\alpha}^{A}:=U_{\alpha} \times \operatorname{Spec}(A)$ and liftings $g_{i \alpha}^{B}$ : $G_{i \alpha}^{B} \longrightarrow M_{i \alpha}^{B}$. By Lemma $\mathrm{C}$ we get extensions $E\left(g_{i \alpha}^{B}\right)$ that glue together over $U_{\alpha \beta}=U_{\alpha} \cap U_{\beta}$ to give global extensions

$$
0 \longrightarrow \mathcal{E}_{i} \longrightarrow E\left(\left\{g_{i \alpha}^{B}\right\}\right) \longrightarrow \mathcal{Q}_{n-i} \longrightarrow 0
$$

Since $\operatorname{Ext}^{1}\left(\mathcal{E}_{i}, \mathcal{Q}_{n-i}\right)=0$ for $i=1, \ldots, n-1$, all the above global extensions split, so the same is true for the affine ones and we can choose liftings $g_{i \alpha}^{B}$ such that $Q_{i \alpha}^{B}$ are flat over $\operatorname{Spec}(B)$ and $Q_{i \alpha}^{B} \rightarrow Q_{i-1 \alpha}^{B}$ for all $i=2, \ldots, n-1$. Over each $U_{\alpha \beta}$ we have two different liftings, hence by Remark D we get an element $\phi_{\alpha \beta} \in K_{\alpha \beta} \otimes J .\left\{\phi_{\alpha \beta}\right\}$ is a cocycle, so it gives a class in $H^{1}(X, \mathcal{K})$, which vanishes by assumption. Therefore $\phi_{\alpha \beta}=\phi_{\alpha}-\phi_{\beta}$, with $\phi_{\alpha} \in K_{\alpha} \otimes J$. Lift $\phi_{\alpha}$ to $\phi_{\alpha}^{B} \in \bigoplus_{i=1}^{n-1} \operatorname{Hom}\left(G_{i \alpha}^{B}, Q_{i \alpha}^{B} \otimes J\right)$. The new maps $\bar{g}_{i \alpha}^{B}=g_{i \alpha}^{B}-\phi_{\alpha}^{B}$ give local liftings that patch together.

Proof of Theorem 1.2. By the previous proposition it suffices to show that for every sequence of successive quotient sheaves

$$
V_{\mathbb{P}^{1}}^{*} \stackrel{p_{n-1}}{\rightarrow} \mathcal{Q}_{n-1} \stackrel{p_{n-2}}{\rightarrow} \mathcal{Q}_{n-2} \rightarrow \ldots \stackrel{p_{1}}{\rightarrow} \mathcal{Q}_{1} \rightarrow 0
$$

such that $\chi\left(\mathbb{P}^{1}, \mathcal{Q}_{i}(m)\right)=(m+1) i+d_{n-i}$, we have $\operatorname{Ext}^{1}\left(\mathcal{E}_{i}, \mathcal{Q}_{n-i}\right)=0$, for $i=$ $1, \ldots, n-1$, and $H^{1}\left(\mathbb{P}^{1}, \mathcal{K}\right)=0$, with $\mathcal{K}$ as above. Since the sheaves $\mathcal{E}_{i}$ are torsionfree on a smooth curve, it follows that they are locally-free and we can identify $\operatorname{Ext}^{1}\left(\mathcal{E}_{i}, \mathcal{Q}_{n-i}\right)$ with $H^{1}\left(\mathbb{P}^{1}, \mathcal{Q}_{n-i} \otimes \mathcal{E}_{i}^{*}\right)$. Since there is a surjection

$$
H^{1}\left(\mathbb{P}^{1}, V_{\mathbb{P}^{1}}^{*} \otimes \mathcal{E}_{i}^{*}\right) \longrightarrow H^{1}\left(\mathbb{P}^{1}, \mathcal{Q}_{n-i} \otimes \mathcal{E}_{i}^{*}\right) \longrightarrow 0
$$

it is enough to prove that $H^{1}\left(\mathbb{P}^{1}, \mathcal{E}_{i}^{*}\right)=0$. Note that the map $j^{*}: V_{\mathbb{P}^{1}} \longrightarrow \mathcal{E}_{i}^{*}$, obtained by dualizing the sheaf inclusion, is generically surjective.

Next we consider the map

$$
V^{*} \otimes V \otimes \mathcal{O}_{\mathbb{P}^{1}}=\mathcal{H}_{o m_{\mathcal{O}^{1}}}\left(V_{\mathbb{P}^{1}}^{*}, V_{\mathbb{P}^{1}}^{*}\right) \longrightarrow \bigoplus_{i=1}^{n-1} \mathcal{H o m}_{\mathcal{O}_{\mathbb{P}}}\left(\mathcal{E}_{i}, \mathcal{Q}_{n-i}\right)
$$


given by

$$
\psi \rightarrow\left\{p_{n-i} \circ \psi \circ j_{i}\right\}_{i=1}^{n-1} .
$$

One can easily check that the above map factors through $\mathcal{K}$, and moreover, it is generically surjective onto $\mathcal{K}$. Therefore, we can conclude the two desired vanishing results from the easy

Lemma $\mathbf{F}$. Let $\mathcal{F}$ be a coherent sheaf on $\mathbb{P}^{1}$ such that there exists a generically surjective map from a trivial bundle on $\mathbb{P}^{1}$ to $\mathcal{F}$. Then $H^{1}\left(\mathbb{P}^{1}, \mathcal{F}\right)=0$.

A standard computation using exact sequences and Riemann-Roch shows that

$$
h^{0}\left(\mathbb{P}^{1}, \mathcal{K}\right)=\frac{n(n-1)}{2}+2 \sum_{i=1}^{n-1} d_{i}
$$

By Proposition E, all irreducible components of $\mathcal{H}_{\bar{d}}$ are smooth, of dimension $\mathcal{D}:=\frac{n(n-1)}{2}+2 \sum_{i=1}^{n-1} d_{i}$. In particular $H_{\bar{d}}$ is an open subset in $\mathcal{H} \mathcal{Q}_{\bar{d}}$.

As for the irreducibility, since we have already shown smoothness, it is enough to prove that $\mathcal{H} \mathcal{Q}_{\bar{d}}$ is connected. First notice that, by Theorem 2.3, successive quotients which do not correspond to morphisms to $F$ vary in families of (base) dimension strictly less than $\mathcal{D}$. (Without knowing the irreducibility of $\mathcal{H} \mathcal{Q}_{\bar{d}-\bar{e}}$, the proof of 2.3 shows only that $\mathcal{U}_{\bar{e}}$ has pure dimension; of course, this does not affect the above conclusion.) Therefore, any connected component will intersect $H_{\bar{d}}$ non-trivially and it suffices to show that $H_{\bar{d}}$ is connected.

Fix $t \in \mathbb{P}^{1}$ and let $\Phi: H_{\bar{d}} \rightarrow F$ be the "evaluation at $t$ " morphism $\Phi([f])=f(t)$. Pick $x \in F$ and $g \in S L(n, \mathbb{C})$. Translation by $g$ induces an isomorphism between the fibres $\Phi^{-1}(x)$ and $\Phi^{-1}(g x)$, given by $[f] \rightarrow[g f]$. Since $S L(n, \mathbb{C})$ acts transitively on $F$, it follows that all the fibres of $\Phi$ are isomorphic. Each fibre can be viewed as the moduli space of based holomorphic maps $\mathbb{P}^{1} \rightarrow F$, with fixed multidegree. By [MM, Corollary 5.19] $\Phi^{-1}(x)$ is connected, hence $H_{\bar{d}}$ is connected as well.

\section{REFERENCES}

[Bat] V.V. Batyrev, Quantum cohomology rings of toric varieties, Astérisque 218 (1991), 9-34.

[Beh] K. Behrend, Gromov-Witten invariants in algebraic geometry, Invent. Math. 127(3) (1997), 601-617. CMP 97:07

[BM] K. Behrend and Y. Manin, Stacks of stable maps and Gromov-Witten invariants, Duke Math. Journal 85 (1996), 1-60. CMP 97:02

[BGG] I. N. Bernstein, I. M. Gelfand, and S. I. Gelfand, Schubert cells and cohomology of the space $G / P$, Russian Math. Surveys 28 (1973), 1-26. MR 55:2941

[Be1] A. Bertram, Towards a Schubert calculus for maps from a Riemann surface to a Grassmannian, Internat. J. Math. 5 (1995), 811-825. MR 96h:14070

[Be2] — Quantum Schubert calculus, Adv. Math. (to appear). CMP 97:14

[BDW] A. Bertram, G. Daskalopoulos, and R. Wentworth, Gromov invariants for holomorphic maps from Riemann surfaces to Grassmannians, J. Amer. Math. Soc. 9 (1996), 529-571. MR 96f: 14066

[Bo] A. Borel, Sur la cohomologie des espaces fibrés principaux et des espaces homogènes des groupes de Lie compacte, Ann. of Math. (2) 57 (1953), 115-207. MR 14:490e

[C-F1] I. Ciocan-Fontanine, Quantum cohomology of flag varieties, Internat. Math. Res. Notices, no. 6 (1995), 263-277. MR 96h:14071

[C-F2] The quantum cohomology ring of flag varieties, University of Utah Ph.D.Thesis (1996). CMP 98:06

$[\mathrm{CM}]$ B. Crauder and R. Miranda, Quantum cohomology of rational surfaces, in The moduli space of curves, R. Dijkgraaf, C. Faber and G. van der Geer, eds., Birkhäuser, 1995, pp. 33-80. MR 97i:14033 
[D] M. Demazure, Désingularization des variétés de Schubert généralisée, Ann. Scient. École Normale Sup. 7 (1974), 53-88. MR 50:7174

[E] C. Ehresmann, Sur la topologie des certaines espaces homogènes, Ann. of Math. 35 (1934), 396-443.

[FGP] S. Fomin, S. Gelfand, and A. Postnikov, Quantum Schubert polynomials, J. Amer. Math. Soc. 10 (1997), 565-596. MR 98d:14063

[F1] W. Fulton, Flags, Schubert polynomials, degeneracy loci and determinantal formulas, Duke Math. Journal 65 (1991), 381-420. MR 93e:14007

[FP] W. Fulton and R. Pandharipande, Notes on stable maps and quantum cohomology, in Proceedings of the 1995 AMS Summer Institute in Santa Cruz (to appear). CMP 98:07

[G] A. Givental, Equivariant Gromov-Witten invariants, Internat. Math. Res. Notices, no. 13 (1996), 613-663. MR 97e:14015

[GK] A. Givental and B. Kim, Quantum cohomology of flag manifolds and Toda lattices, Comm. Math. Phys. 168 (1995), 609-641. MR 96c:58027

[Gr] A. Grothendieck, Techniques de construction et théorèmes d'existence en géometrie algébrique IV: Les schémas de Hilbert, Séminaire Bourbaki 221 (1960/61). CMP 98:09

[H] R. Hartshorne, Algebraic Geometry, Springer Verlag, 1977. MR 57:3116

[Ki1] B. Kim, Quot schemes for flags and Gromov invariants for flag varieties, preprint (1995).

[Ki2] On equivariant quantum cohomology, Internat. Math. Res. Notices, no. 17 (1996), 841-851. CMP 97:04

[Ki3] Q Quantum cohomology of flag manifolds $G / B$ and quantum Toda lattices, preprint (1996).

[Kl] S. Kleiman, The transversality of a general translate, Compositio Math. 28 (1974), 287297. MR 50:13063

[Kol] J. Kollár, Rational curves on algebraic varieties, Springer Verlag, 1996. MR 98c:14001

[KM] M. Kontsevich and Y. Manin, Gromov-Witten classes, quantum cohomology and enumerative geometry, Comm. Math. Phys. 164 (1994), 525-562. MR 95i:14049

[Kuz] A. Kuznetsov, Laumon's resolution of Drinfeld's compactification is small, Math. Research Letters 4 (1997), 349-364. MR 98d:14022

[LS1] A. Lascoux and M.-P. Schützenberger, Polynômes de Schubert, C.R. Acad. Sci. Paris 294 (1982), 447-450. MR 83e:14039

[LS2] Symmetry and flag manifolds, in Invariant Theory, F. Gherardelli ed., Lecture Notes in Math., vol. 996, Springer, Berlin, 1983, pp. 118-144. MR 85e:14073

[Lau1] G. Laumon, Un anlogue global du cône nilpotent, Duke Math. Journal 57 (1988), 647-671. MR 90a:14012

[Lau2] _ Faisceaux automorphes liés aux sèries d'Eisenstein, in Automorphic forms, Shimura varieties and L-functions, L. Clozel and J.S. Milne eds., vol. 1, Academic Press, 1990, pp. 227-281. MR 91k:11106

[LT1] J.Li and G. Tian, Quantum cohomology of homogeneous varieties, preprint (1995).

[LT2] - Virtual moduli cycles and Gromov-Witten invariants, preprint (1996).

$[\mathrm{MM}]$ B. Mann and J. Milgram, On the moduli space of $S U(n)$ monopoles and holomorphic maps to flag manifolds, J. Differential Geom. 38 (1993), 39-103. MR 95c:58031

[Mo] D. Monk, The geometry of flag manifolds, Proc. London Math. Soc.(3) 9 (1959), 253-286. MR 21:5641

[Mu D. Mumford, Lectures on curves on an algebraic surface, Princeton Univ. Press, Princeton, NJ, 1966. MR 35:187

[QR] Z. Qin and Y. Ruan, Quantum cohomology of projective bundles over $\mathbb{P}^{n}$, Trans. Amer. Math. Soc. (to appear). CMP 97:05

[RT] Y. Ruan, G. Tian, A mathematical theory of quantum cohomology, J. Differential Geom. 42 (1995), 259-367. MR 96m:58033

[ST] B. Siebert, G. Tian, On quantum cohomology of Fano manifolds and a formula of Vafa and Intriligator, preprint (1994). MR 90b:81080

[W] E. Witten, Topological sigma model, Commun. Math. Phys. 118 (1988), 411-449.

Department of Mathematics, University of Utah, Salt Lake City, Utah 84112 Current address: Department of Mathematics, Northwestern University, 2033 Sheridan Road, Evanston, Illinois 60208-2730

E-mail address: ciocan@math.nwu.edu 